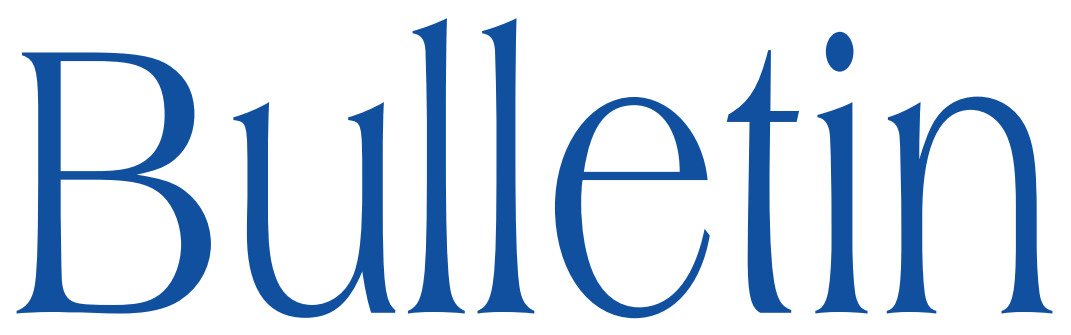

de la SOCIÉTÉ MATHÉMATIQUE DE FRANCE

\title{
FONCTION Z ÊTA DES HAUTEURS ASSOCIÉE À UNE CERTAINE SURFACE CUBIQUE
}

Régis de la Bretèche \& Sir Peter Swinnerton-Dyer

\section{Tome 135}

Fascicule 1

2007 


\title{
FONCTION ZÊTA DES HAUTEURS ASSOCIÉE À UNE CERTAINE SURFACE CUBIQUE
}

\author{
PAR Régis de la Bretèche \& Sir Peter Swinnerton-Dyer
}

\begin{abstract}
RÉsumé. - L'objet de cet article est d'obtenir une formule pour la fonction zêta des hauteurs classique à partir de la fonction zêta des hauteurs multiple de La Bretèche, et d'utiliser cette formule pour prolonger de manière méromorphe la fonction zêta des hauteurs. En particulier, il est montré que celle-ci peut être prolongée au demi-plan $\left\{s \in \mathbb{C}: \Re e s>\frac{3}{4}\right\}$ et que la frontière naturelle de son domaine naturel de méromorphie est $\left\{s \in \mathbb{C}: \Re e s=\frac{3}{4}\right\}$.

Abstract (Height zeta function of a cubic surface). - The object of this article is to obtain a formula for the classical height zeta function of $X_{0}^{3}=X_{1} X_{2} X_{3}$ in terms of the multiple height zeta function of La Bretèche, and to use that formula to find the meromorphic continuation of the height zeta function. In particular, it will be shown that the height zeta function can be meromorphic continued in $\left\{s \in \mathbb{C}: \Re e s>\frac{3}{4}\right\}$ and its natural boundary is $\left\{s \in \mathbb{C}: \Re e s=\frac{3}{4}\right\}$.
\end{abstract}

Texte reçu le 17 janvier 2006, révisé le 25 juillet 2006

Régis de la Bretèche, Université Paris 7 - Denis Diderot, Institut de Mathématiques de Jussieu, UMR 7586, Case 7012, 2, place Jussieu, 75251 Paris Cedex 05 (France)

E-mail : breteche@math.jussieu.fr

Sir Peter Swinnerton-Dyer, Isaac Newton Institute, 20, Clarkson Road, Cambridge CB3 0EH (England) • E-mail : H.P.F.Swinnerton-Dyer@dpmms.cam.ac.uk

Classification mathématique par sujets (2000). - 11G35, 14G05, 14G10.

Mots clefs. - Conjecture de Manin, surfaces cubiques, frontière naturelle du domaine naturel de méromorphie, utilisation de l'hypothèse de Riemann, formule de Perron. 


\section{Introduction}

De nombreux auteurs (voir $[2,7,8,9]$ ) ont étudié l'asymptotique du nombre $N(B)$ des points rationnels $P$ de hauteur $H(P) \leqslant B$ sur la surface cubique définie par

$$
X_{0}^{3}=X_{1} X_{2} X_{3}
$$

en dehors des trois droites définies par $X_{0}=0$. Ici, la hauteur $H(P)$ lorsque $P$ est représenté par un quadruplet d'entiers $x_{0}, x_{1}, x_{2}, x_{3}$ de pgcd égal à 1 est définie par

$$
H(P)=\max _{j}\left|x_{j}\right| .
$$

Le meilleur résultat a été obtenu par le premier auteur [2] utilisant des méthodes d'analyse complexe analogue à celle intervenant dans la démonstration du théorème des nombres premiers. Il a montré qu'il existe un polynôme $Q$ de degré 6 et une constante $c>0$ tels que l'on ait, pour $B$ tendant vers l'infini, l'estimation

$$
N(B)=B Q(\log B)+O\left(B^{7 / 8} \exp \left\{-c(\log B)^{3 / 5}(\log \log B)^{-1 / 5}\right\}\right) .
$$

De plus le coefficient dominant est égal à

$$
\frac{1}{6 !} \prod_{p}\left\{\left(1-\frac{1}{p}\right)^{7}\left(1+\frac{7}{p}+\frac{1}{p^{2}}\right)\right\} .
$$

De récentes conjectures concernant le nombre de points rationnels [11] rendent souhaitable d'obtenir le terme suivant de l'estimation de $N(B)$. Le second auteur conjecture dans [11] qu'il existe un deuxième terme dans l'estimation de $N(B)$ pour les surfaces cubiques non-singulières qui devrait ressembler à celui qui apparaît dans la formule explicite du théorème de nombres premiers.

L'exposant $\frac{9}{11}$ apparaissant dans (1.5) est difficile à prévoir. Il est sans doute lié à la nature de la singularité de la cubique considérée, mais nous pensons qu'il est plausible de l'attribuer au choix de la hauteur. Un choix plus canonique d'un certain point de vue de la hauteur peut être trouvé dans [12]. Pour celle-ci, on peut remplacer (1.4) par

$$
N(B)=B Q_{1}(\log B)+O\left(B^{1 / 2+\epsilon}\right)
$$

où $Q_{1}$ est un polynôme de degré 5 et le terme d'erreur est encore relié aux zéros de la fonction zêta de Riemann; mais ici aussi il y a des complications qui apparaissent de manière quelque peu non naturelle.

Puisque les conjectures de [11] ne concernent que les cubiques non-singulières, cela ne permet pas de faire des prédictions sur la valeur de cet exposant.

Les fonctions zêta des hauteurs associées à certaines surfaces de del Pezzo singulières de degré 4 ont déjà été calculéees dans [3, 4, 5]. Elles jouent un role 
clé dans la démonstration d'une estimation précise dans la conjecture de Manin et l'étude de la taille du terme d'erreur. De plus, elles recellent en général plus d'informations que les formules asymptotiques.

Nous étudions ici les propriétés analytiques de la fonction zêta des hauteurs $Z(s)$ associée à (1.1) et en particulier le plus grand domaine où la fonction $Z(s)$ peut être prolongée de manière méromorphe.

La fonction zêta des hauteurs $Z(s)$ associée à (1.1) sur lequel on compte est définie par

$$
Z(s)=\sum_{P} \frac{1}{H(P)^{s}}
$$

où la somme est prise sur les points rationnels en dehors de la réunion des trois droites d'équation $X_{0}=0$.

THÉORÈmE 1. - La fonction $Z(s)$ admet un prolongement méromorphe dans le demi-plan $\left\{s \in \mathbb{C}: \operatorname{Re} s>\frac{3}{4}\right\}$ et sa frontière naturelle d'analycité est la droite $\left\{s \in \mathbb{C}: \operatorname{Re} s=\frac{3}{4}\right\}$.

Il est démontré dans [6] que les fonctions zêtas des hauteurs associés aux espaces projectifs $\mathbb{P}_{n}(Q)$ et à l'éclaté du plan projectif en un point sur $\mathbb{Q}$ sont prolongeables en des fonctions méromorphes sur $\mathbb{C}$. À notre connaissance, c'est le seul autre exemple connu où le domaine naturel de méromorphie est entièrement déterminé.

L'estimation (1.5) avec son terme d'erreur semblable à celui qui apparaît dans le théorème des nombres premiers laisse deviner l'importance de la répartition des zéros de $\zeta$ dans la taille du terme d'erreur.

La formule (1.4) permet d'obtenir une évaluation de $N(B)$ sous l'hypothèse de Riemann usuelle. Les pôles de $Z(s)$ dans $\{s \in \mathbb{C}: \operatorname{Re} s \in] \frac{4}{5}, 1[\}$ sont alors $\frac{9}{11}$ et les $\frac{3}{4}+\frac{1}{8} \rho$ où les $\rho$ décrivent les zéros de $\zeta$ de partie réelle $\frac{1}{2}$.

ThÉORÈme 2. - Soit $\varepsilon>0$. Sous l'hypothèse de Riemann, il existe une constante $\gamma>0$ telle que nous avons lorsque $B$ tendant vers l'infini l'estimation

$$
N(B)=B Q(\log B)+\gamma B^{9 / 11}+O\left(B^{13 / 16+\varepsilon}\right) .
$$

Si de plus tous les zéros de $\zeta$ sont simples, nous avons

$$
N(B)=B Q(\log B)+\gamma B^{9 / 11}+\lim \sum_{|\tau| \leqslant R} \beta_{\tau} B^{13 / 16+i \tau / 8}+O\left(B^{4 / 5+\varepsilon}\right)
$$

où les $\frac{1}{2}+i \tau$ parcourent les zéros de la fonction zêta, les $\beta_{\tau}$ ne dépendent que de $\tau$, et la limite est prise lorsque $R \rightarrow+\infty$ avec la condition

$$
|\zeta(\sigma+i R)|>R^{-1 / \log \log \log R} \quad\left(\frac{1}{2} \leqslant \sigma<1\right) .
$$


REMARQUES. - Il n'y a aucune raison de penser que $\zeta$ admet un zéro multiple. Mais si $\frac{1}{2}+i \tau$ est un zéro d'ordre $r>1$ de $\zeta$, il suffit dans (1.5) de multiplier le terme correspondant par $Q_{\tau}(\log B)$ où $Q_{\tau}$ est un polynôme de degré $r-1$.

L'ensemble des $R$ satisfaisant (1.6) est non borné. En effet, le théorème 14.16 de [14] affirme que, pour un choix convenable de $A$, tout intervalle $[T, T+1]$ avec $T \geqslant 2$ contient un $t$ tel que

$$
|\zeta(\sigma+i t)|>t^{-A / \log \log t} \quad\left(\frac{1}{2} \leqslant \sigma<1\right)
$$

ce qui est plus fort que (1.6).

Nous renvoyons à la formule (4.9) pour une expression explicite de $\gamma$ et à (4.12) pour une de $\beta_{\tau}$.

Remerciements. - Ce travail a été réalisé lorsque le premier auteur faisait partie de l'équipe Arithmétique et géométrie algébrique du département de Mathématiques de l'Université d'Orsay. Nous remercions aussi Jordan Ellenberg qui nous a conseillé de déterminer le signe de la constante $\gamma$ apparaissant au théorème 2 .

\section{Démonstration}

2.1. Préliminaires. - Dans une première étape, nous démontrons une identité qui peut être vue comme une variante de la formule de Perron; celle-ci est donnée au lemme 2. Avant cela, nous énonçons un lemme qui nous permettra de justifier les différentes manipulations en montrant la convergence absolue de toutes les intégrales multiples que nous rencontrerons par la suite.

Nous n'utiliserons seulement que le cas $n=0$ du lemme 1 , mais l'énoncé du résultat général servira dans la démonstration par récurrence.

Lemme 1. - Soient $\alpha, \gamma$ des réels et $r, n$ des entiers tels que $0 \leqslant \alpha<1, \gamma>0$, $r>0, n \geqslant 0$. Pour tout $t$ l'intégrale

$$
\int_{-\infty}^{\infty} \cdots \int_{-\infty}^{\infty} \frac{T^{\alpha}\left\{\log \left(2+\left|t-t_{1}-\cdots-t_{r}\right|\right)\right\}^{n} \mathrm{~d} t_{1} \cdots \mathrm{d} t_{r}}{\left(\gamma+\left|t_{1}\right|\right) \cdots\left(\gamma+\left|t_{r}\right|\right)\left(\gamma+\left|t-t_{1}-\cdots-t_{r}\right|\right)}
$$

où $T=\max \left(\left|t_{1}\right|, \ldots,\left|t_{r}\right|\right)$ est convergente et uniformément bornée.

Démonstration. - Nous raisonnons par récurrence sur $r$. Il est clair que, pour des raisons de symétrie, on peut remplacer dans (2.1) le terme $T^{\alpha}$ par $\left|t_{1}\right|^{\alpha}$. Nous majorons l'intégrale sur $t_{r}$ pour laquelle nous devons considérer

$$
\int_{-\infty}^{\infty}\left|t_{r}\right|^{\alpha_{r}} \frac{\left\{\log \left(2+\left|\tau-t_{r}\right|\right)\right\}^{n} \mathrm{~d} t_{r}}{\left(\gamma+\left|t_{r}\right|\right)\left(\gamma+\left|\tau-t_{r}\right|\right)}
$$


où nous avons utilisé la notation

$$
\tau= \begin{cases}t-t_{1}-\cdots-t_{r-1} & \text { avec } \alpha_{r}=\alpha \text { si } r=1 \\ 0 & \text { sinon. }\end{cases}
$$

Puisque nous pouvons changer les signes de $t$ et de tous les $t_{j}$, nous pouvons supposer $\tau \geqslant 0$. Nous scindons le domaine d'intégration de (2.2) en quatre.

- Lorsque $t_{r} \geqslant 2 \tau$ ou $t_{r} \leqslant-\tau$, l'intégrande est majorée par

$$
O\left(\left\{\log \left(2+\left|t_{r}\right|\right)\right\}^{n} /\left(\gamma+\left|t_{r}\right|\right)^{2-\alpha_{r}}\right)
$$

de sorte que la contribution à l'intégrale (2.2) est $O\left(\{\log (2+\tau)\}^{n} /(\gamma+\tau)^{1-\alpha_{r}}\right)$.

- Dans $2 \tau \geqslant t_{r} \geqslant \frac{1}{2} \tau$, l'intégrande est majorée par

$$
O\left(\{\log (2+\tau)\}^{n} /(\gamma+\tau)^{1-\alpha_{r}}\left(\gamma+\left|\tau-t_{r}\right|\right)\right)
$$

et la contribution à l'intégrale $(2.2)$ est $O\left(\{\log (2+\tau)\}^{n+1} /(\gamma+\tau)^{1-\alpha_{r}}\right)$.

- Des arguments similaires permettent de la même manière de traiter l'intervalle $\frac{1}{2} \tau \geqslant t_{r} \geqslant-\tau$.

Nous avons donc montré que l'intégrale de (2.2) est

$$
O\left(\{\log (2+\tau)\}^{n+1} /(\gamma+\tau)^{1-\alpha_{r}}\right) .
$$

Notant temporairement (2.1) par $I_{n, r}(t)$, nous avons établi que $I_{n, r}(t)$ est convergente lorsque $r \geqslant 1$ si $I_{n+1, r-1}(t)$ l'est aussi, ce qui permet d'itérer la récurrence, et que $I_{n, 1}(t)$ est borné ce qui permet d'initialiser la récurrence.

Pour des nombres complexes $s_{j}$, considérons une série de Dirichlet à plusieurs variables

$$
f\left(s_{0}, \ldots, s_{r}\right)=\sum a\left(n_{0}, \ldots, n_{r}\right) n_{0}^{-s_{0}} \ldots n_{r}^{-s_{r}}
$$

absolument convergente lorsque $\operatorname{Re} s_{0}>c_{0}^{\prime}, \ldots, \operatorname{Re} s_{r}>c_{r}^{\prime}$, où $c_{j}^{\prime} \geqslant 0$ pour tout $j$. Écrivant $m=\max \left(n_{0}, \ldots, n_{r}\right)$, nous associons à $f$ la série de Dirichlet

$$
Z(s)=\sum a\left(n_{0}, \ldots, n_{r}\right) m^{-s},
$$

qui est absolument convergente dans $\operatorname{Re} s>c_{0}^{\prime}+\cdots+c_{r}^{\prime}$.

LEMme 2. - Soient $c_{j}>c_{j}^{\prime}$ lorsque $1 \leqslant j \leqslant r$ et $s$ un nombre complexe satisfaisant $\operatorname{Re} s>c_{0}^{\prime}+c_{1}+\cdots+c_{r}$. Alors $Z(s)$ est égale à

$$
\frac{1}{(2 \pi i)^{r}} \int_{c_{1}-i \infty}^{c_{1}+i \infty} \cdots \int_{c_{r}-i \infty}^{c_{r}+i \infty} \frac{s f\left(s-s_{1}-\cdots-s_{r}, s_{1}, \ldots, s_{r}\right)}{s_{1} \cdots s_{r}\left(s-s_{1}-\cdots-s_{r}\right)} \mathrm{d} s_{1} \cdots \mathrm{d} s_{r} .
$$

REMARQUe. - Le membre de droite de (2.3) n'est pas symétrique en les variables $s_{j}$. Il existe des formules symétriques pour $Z(s)$ en fonction de $f$, mais elles sont plus compliquées et moins pratiques. 
Démonstration. — Commençons par considérer le cas

$$
f\left(s_{0}, \ldots, s_{r}\right)=n_{0}^{-s_{0}} \cdots n_{r}^{-s_{r}} .
$$

Nous partons de la formule classique, valable pour $c_{1}>0$,

$$
h(x)=\frac{1}{2 \pi i} \int_{c_{1}-i \infty}^{c_{1}+i \infty} \frac{x^{s_{1}}}{s_{1}} \mathrm{~d} s_{1}= \begin{cases}1 & \text { si } x>1, \\ \frac{1}{2} & \text { si } x=1, \\ 0 & \text { si } x<1 .\end{cases}
$$

Nous raisonnons par récurrence sur $r$. Lorsque $r=1$, le membre de droite de (2.3) est

$$
\frac{1}{2 \pi i} \int_{c_{1}-i \infty}^{c_{1}+i \infty} \frac{s n_{0}^{s_{1}-s} n_{1}^{-s_{1}}}{s_{1}\left(s-s_{1}\right)} \mathrm{d} s_{1}
$$

et s'écrit sous la forme

$$
\begin{aligned}
& =\frac{n_{0}^{-s}}{2 \pi i} \int_{c_{1}-i \infty}^{c_{1}+i \infty} \frac{\left(n_{0} / n_{1}\right)^{s_{1}}}{s_{1}} \mathrm{~d} s_{1}+\frac{n_{1}^{-s}}{2 \pi i} \int_{c_{1}-i \infty}^{c_{1}+i \infty} \frac{\left(n_{1} / n_{0}\right)^{s-s_{1}}}{s-s_{1}} \mathrm{~d} s_{1} \\
& =n_{0}^{-s} h\left(n_{0} / n_{1}\right)+n_{1}^{-s} h\left(n_{1} / n_{0}\right)=\left\{\max \left(n_{0}, n_{1}\right)\right\}^{-s}
\end{aligned}
$$

avec $\operatorname{Re} s>c_{0}^{\prime}+c_{1}$.

Lorsque $r>1$, nous utilisons ce résultat avec $s^{\prime}=s-s_{2}-\cdots-s_{r}$ à la place de $s$ pour intégrer en la variable $s_{1}$; l'expression (2.3) de $f$ lorsque $f$ est donné par (2.4) est égale à

$$
\frac{1}{(2 \pi i)^{r-1}} \int_{c_{2}-i \infty}^{c_{2}+i \infty} \cdots \int_{c_{r}-i \infty}^{c_{r}+i \infty} \frac{s\left\{\max \left(n_{0}, n_{1}\right)\right\}^{-s^{\prime}} n_{2}^{-s_{2}} \cdots n_{r}^{-s_{r}}}{s^{\prime} s_{2} \cdots s_{r}} \mathrm{~d} s_{2} \cdots \mathrm{d} s_{r} .
$$

C'est exactement l'expression du membre de droite de (2.3) avec un variable en moins; une itération de ce procédé fournit alors la valeur recherchée

$$
\left\{\max \left(n_{0}, n_{1}, \ldots, n_{r}\right)\right\}^{-s} \text {. }
$$

Cela prouve le cas particulier (2.4). Le cas général s'en déduit par interversion de sommation.

2.2. Séries de Dirichlet à plusieurs variables. - Soit $U$ l'ouvert de la surface projective $X_{0}^{3}=X_{1} X_{2} X_{3}$ défini par $X_{0} \neq 0$. Nous préparons l'application du lemme 2. Nous notons

$$
f\left(s_{1}, s_{2}, s_{3}\right)=\sum x_{1}^{-s_{1}} x_{2}^{-s_{2}} x_{3}^{-s_{3}}
$$

où la somme est prise sur les triplets $x_{1}, x_{2}, x_{3}$ d'entiers positifs de pgcd égal à 1 tels que le produit $x_{1} x_{2} x_{3}$ soit un cube. Alors la fonction zêta des hauteurs $Z(s)$ associée à l'ouvert $U$ est donnée par

$$
Z(s)=4 \sum\left\{\max \left(x_{1}, x_{2}, x_{3}\right)\right\}^{-s} .
$$


Notons

$$
\phi\left(z, s_{1}, s_{2}, s_{3}\right)=\frac{1+\sum\left(1-z^{3 s_{i}}\right) z^{2 s_{j}+s_{k}}-z^{3 s_{1}+3 s_{2}+3 s_{3}}}{\left(1-z^{3 s_{1}}\right)\left(1-z^{3 s_{2}}\right)\left(1-z^{3 s_{3}}\right)}
$$

où la somme est prise sur les six permutations $i, j, k$ de $1,2,3$; cette convention sera adoptée jusqu'à la fin du prochain paragraphe. Le premier auteur a montré la formule

$$
f\left(s_{1}, s_{2}, s_{3}\right)=\prod_{p} \phi\left(p^{-1}, s_{1}, s_{2}, s_{3}\right)
$$

que nous utilisons pour prolonger $f$. Soit $N>2$. Pour les triplets d'entiers $n_{1}, n_{2}, n_{3}$ avec $0<n_{1}+n_{2}+n_{3} \leqslant N$, on peut déterminer de manière unique les entiers $b\left(n_{1}, n_{2}, n_{3}\right)$ tels que la fonction $\psi_{N}$ définie implicitement par

$$
\phi\left(z, s_{1}, s_{2}, s_{3}\right)=\left\{\prod\left(1-z^{n_{1} s_{1}+n_{2} s_{2}+n_{3} s_{3}}\right)^{-b\left(n_{1}, n_{2}, n_{3}\right)}\right\} \psi_{N}\left(z, s_{1}, s_{2}, s_{3}\right)
$$

s'écrive

$$
\psi_{N}=1+\sum c_{N}\left(n_{1}, n_{2}, n_{3}\right) z^{n_{1} s_{1}+n_{2} s_{2}+n_{3} s_{3}}
$$

où la somme ci-dessus ne contient pas de termes tels que $0<n_{1}+n_{2}+n_{3} \leqslant N$. Pour éviter les cas particuliers, lorsque $N=1,2$ nous écrivons

$$
\begin{aligned}
\psi_{N} & =\left(1-z^{3 s_{1}}\right)\left(1-z^{3 s_{2}}\right)\left(1-z^{3 s_{3}}\right) \phi \\
& =1+\sum\left(1-z^{3 s_{i}}\right) z^{2 s_{j}+s_{k}}-z^{3 s_{1}+3 s_{2}+3 s_{3}} .
\end{aligned}
$$

L'existence et l'unicité des $b\left(n_{1}, n_{2}, n_{3}\right)$ peuvent être démontrées par récurrence sur $N$; pour aller de $N$ à $N+1$ on peut et on doit choisir les $b\left(n_{1}, n_{2}, n_{3}\right)$ avec $n_{1}+n_{2}+n_{3}=N+1$ égaux aux $c_{N}\left(n_{1}, n_{2}, n_{3}\right)$. Il est facile aussi de voir qu'une permutation sur les $n_{j}$ ne change pas la valeur des $c_{N}\left(n_{1}, n_{2}, n_{3}\right)$, que l'on a

$$
c_{N}\left(n_{1}, n_{2}, n_{3}\right)=0 \quad \text { sauf si } 3 \mid\left(n_{1}+n_{2}+n_{3}\right),
$$

et que pour toute permutation $i, j, k$ de $1,2,3$

$$
c_{N}\left(n_{1}, n_{2}, n_{3}\right)=0 \quad \text { lorsque } n_{i}>2\left(n_{j}+n_{k}\right) .
$$

Donc, avec la restriction $n_{1}+n_{2}+n_{3}>3$ dans le cas (2.12), ces propriétés restent vraies pour $b\left(n_{1}, n_{2}, n_{3}\right)$. Pour faciliter le rappel, nous énonçons cela formellement.

LEMME 3. - La valeur $b\left(n_{1}, n_{2}, n_{3}\right)$ est invariante par permutation des arguments. Si $b\left(n_{1}, n_{2}, n_{3}\right) \neq 0$ alors $3 \mid\left(n_{1}+n_{2}+n_{3}\right)$ et toutes les conditions $n_{i} \leqslant 2\left(n_{j}+n_{k}\right)$ sont vérifiées si $n_{1}, n_{2}, n_{3}$ ne prend pas les valeurs $3,0,0$ à permutation près.

REmARque. - Nous remercions l'arbitre de cet article de nous avoir indiqué que de semblables manipulations sont effectuées dans [1]. 
Pour prolonger $Z(s)$ jusqu'à $\operatorname{Re} s>\frac{3}{4}$ nous aurons aussi besoin des relations

$$
b(3,0,0)=b(2,1,0)=1, \quad b(1,1,1)=0,
$$

couplées avec $b(6,3,0)=0$ et

$$
b(4,2,0)=b(4,1,1)=-1, \quad b(3,2,1)=b(2,2,2)=-2,
$$

ou au moins de retenir que ces dernières valeurs sont toutes négatives.

Nous exposons maintenant un moyen simple de calculer les $b\left(n_{1}, n_{2}, n_{3}\right)$. Les membres de droite de (2.7) et (2.9) sont égaux; en prenant le logarithme, en appliquant l'opérateur $z \partial / \partial z$ puis en faisant tendre $N$ vers l'infini nous obtenons l'égalité entre séries formelles

$$
\begin{gathered}
\sum \frac{b\left(n_{1}, n_{2}, n_{3}\right)\left(n_{1} s_{1}+n_{2} s_{2}+n_{3} s_{3}\right) z^{n_{1} s_{1}+n_{2} s_{2}+n_{3} s_{3}}}{1-z^{n_{1} s_{1}+n_{2} s_{2}+n_{3} s_{3}}} \\
=\frac{3 s_{1} z^{3 s_{1}}}{1-z^{3 s_{1}}}+\frac{3 s_{2} z^{3 s_{2}}}{1-z^{3 s_{2}}}+\frac{3 s_{3} z^{3 s_{3}}}{1-z^{3 s_{3}}} \\
\quad+z \frac{\partial}{\partial z} \log \left\{1+\sum\left(1-z^{3 s_{i}}\right) z^{2 s_{j}+s_{k}}-z^{3\left(s_{1}+s_{2}+s_{3}\right)}\right\}
\end{gathered}
$$

qui permet d'en déduire les valeurs des $b\left(n_{1}, n_{2}, n_{3}\right)$. Une version encore plus efficace peut être obtenue en remarquant que le numérateur de $\phi$ dans (2.7) se factorise de la manière suivante

$$
\left(1-z^{s_{1}+s_{2}+s_{3}}\right)\left(1+z^{s_{1}+s_{2}+s_{3}}+\sum z^{2 s_{i}+s_{j}}+z^{2\left(s_{1}+s_{2}+s_{3}\right)}\right),
$$

et en lui appliquant le même procédé.

Si $3 \mid\left(n_{1}+n_{2}+n_{3}\right)$ et si toutes les inégalités $n_{i} \leqslant 2\left(n_{j}+n_{k}\right)$ sont satisfaites, on a alors

$$
b\left(n_{1}, n_{2}, n_{3}\right) \begin{cases}\geqslant 0 & \text { si } n_{1}+n_{2}+n_{3} \text { est impair } \\ \leqslant 0 & \text { si } n_{1}+n_{2}+n_{3} \text { est pair. }\end{cases}
$$

Cette assertion étant inutile dans la suite de la démonstration, nous omettons de la démontrer.

LEMME 4. - Lorsque $N>2$ le produit

$$
\Psi_{N}\left(s_{1}, s_{2}, s_{3}\right)=\prod_{p} \psi_{N}\left(p^{-1}, s_{1}, s_{2}, s_{3}\right)
$$

est absolument convergent et définit une fonction holomorphe dans le domaine défini par les inégalités $\operatorname{Re}\left(2 s_{i}+s_{j}\right)>3 /(N+1)$ prises pour toutes les permutations $i, j, k$ de 1,2,3. De plus, si pour un $\varepsilon>0$ les $s_{\nu}$ satisfont les six conditions

$$
\operatorname{Re}\left(2 s_{i}+s_{j}\right)>3 /(N+1)+\varepsilon
$$

alors $\Psi_{N}$ est borné par un majorant ne dépendant que de $\varepsilon$ et de $N$.

TOME $135-2007-\mathrm{N}^{\mathrm{O}} 1$ 
Remarque. - Ce résultat a été démontré auparavant dans [1]. Afin d'avoir une démonstration autonome, nous détaillons la démonstration.

Démonstration. - Lorsque $s_{1}=s_{2}=s_{3}=1$, la série $\psi_{N}$ est absolument convergente dans $|z|<1$ pour tout $N$ car on l'a construite comme un produit fini de séries absolument convergentes. Donc pour tout $\rho>1$ il existe $A_{N, \rho}$ dépendant uniquement de $\rho$ et de $N$ tel que tous les coefficients de (2.10) satisfassent

$$
\left|c_{N}\left(n_{1}, n_{2}, n_{3}\right)\right|<A_{N, \rho} \rho^{n_{1}+n_{2}+n_{3}} .
$$

Posons $\alpha=\min \left(\operatorname{Re}\left(2 s_{i}+s_{j}\right)\right)$ de sorte que $\alpha>3 /(N+1)+\varepsilon$. Nous déduisons de (2.12) que si $c_{N}\left(n_{1}, n_{2}, n_{3}\right) \neq 0$ alors $n_{1} s_{1}+n_{2} s_{2}+n_{3} s_{3}$ est une combinaison linéaire des six $2 s_{i}+s_{j}$; donc sa partie réelle est au moins égale à $\frac{1}{3} \alpha \nu$ avec $\nu=n_{1}+n_{2}+n_{3}$. Pour tout $\nu>N$ la série (2.10) contient $O\left(\nu^{2}\right)$ termes avec $n_{1}+n_{2}+n_{3}=\nu$; lorsque $|z|<\rho^{-3 / \alpha}$ la série est donc majorée par

$$
1+B_{N, \rho} \sum_{\nu=N+1}^{\infty} \nu^{2}|z|^{\nu / N} \rho^{\nu}=1+O_{N, \rho}\left(\frac{|z|^{\alpha(N+1) / 3} \rho^{N}}{\left(1-\rho|z|^{\alpha / 3}\right)^{3}}\right) .
$$

Puisque $\alpha(N+1) / 3>1$ le produit des $\psi_{N}\left(p^{-1}, s_{1}, s_{2}, s_{3}\right)$ est absolument convergent, et par conséquent définit une fonction holomorphe dans le domaine défini dans le lemme. Puisque la démonstration que $\Psi_{N}$ soit absolument convergent dépend seulement d'une comparaison avec une expression indépendante de $s_{j}$, la dernière assertion du lemme est aussi prouvée.

Corollaire . - La fonction $f\left(s_{1}, s_{2}, s_{3}\right)$ peut être prolongée dans le domaine défini par les inégalités $\operatorname{Re}\left(2 s_{i}+s_{j}\right)>0$ prises pour toutes les permutations $i, j, k$ de $1,2,3$. En particulier, si $s_{1}, s_{2}, s_{3}$ sont des fonctions affines de $s$ alors la fonction de $s$ que définit $f\left(s_{1}, s_{2}, s_{3}\right)$ est méromorphe dans toute bande de la forme $\sigma^{\prime}<\operatorname{Re} s<\sigma^{\prime \prime}$ dans laquelle $\operatorname{Re}\left(2 s_{i}+s_{j}\right)>0$.

Démonstration. - Grâce à (2.9), nous avons lorsque $N>2$

$$
f\left(s_{1}, s_{2}, s_{3}\right)=\left\{\prod\left(\zeta\left(n_{1} s_{1}+n_{2} s_{2}+n_{3} s_{3}\right)\right)^{b\left(n_{1}, n_{2}, n_{3}\right)}\right\} \Psi_{N}\left(s_{1}, s_{2}, s_{3}\right),
$$

où le produit est pris pour tous les triplets $n_{1}, n_{2}, n_{3}$ qui vérifient les conditions $0<n_{1}+n_{2}+n_{3} \leqslant N$. En prenant $N$ arbitrairement grand, nous obtenons grâce au lemme 4 le prolongement de $f$ dans le domaine désiré. 
2.3. Prolongement de la fonction zêta des hauteurs. - Pour tous réels positifs $c_{2}, c_{3}$, considérons

$$
J\left(c_{2}, c_{3}\right)=\frac{1}{(2 \pi i)^{2}} \int_{c_{2}-i \infty}^{c_{2}+i \infty} \int_{c_{3}-i \infty}^{c_{3}+i \infty} \frac{s f\left(s-s_{2}-s_{3}, s_{2}, s_{3}\right)}{s_{2} s_{3}\left(s-s_{2}-s_{3}\right)} \mathrm{d} s_{2} \mathrm{~d} s_{3} .
$$

Pour raccourcir les formules nous utiliserons la notation $s_{1}=s-s_{2}-s_{3}$. Grâce au lemme 2 et à (2.6), on a

$$
Z(s)=4 J\left(c_{2}, c_{3}\right)
$$

pour $c_{2}>\frac{1}{3}, c_{3}>\frac{1}{3}$ et $\operatorname{Re} s>\frac{1}{3}+c_{2}+c_{3}$; cela fournit déjà une description de $Z(s)$ dans $\operatorname{Re} s>1$. Pour prolonger $J=J\left(c_{2}, c_{3}\right)$ nous avons besoin de déplacer les contours d'intégration de $s_{2}$ et de $s_{3}$ vers la gauche. Pour simplifier, nous décrivons ce procédé en disant que nous déplaçons $s_{3}$ dans $J$ de $\operatorname{Re} s_{3}=\gamma$ à $\operatorname{Re} s_{3}=\gamma^{\prime}$. Lorsque nous faisons cela pour $s$ appartenant dans une bande verticale, nous devons contrôler $\operatorname{Re} s$ de sorte que nous puissions savoir quels pôles - s'écrivant en fonction de $s, s_{2}$ - le contour de $s_{3}$ croise; alors $J$ devient une somme d'intégrales sur $s_{2}$ qui correspondent aux résidus associés aux différents pôles avec une valeur de $J$ ayant des arguments différents. Le premier exemple de ce procédé est fourni en (2.19); et sa pertinence provient du fait qu'on peut montrer que le membre de droite peut être défini dans une bande débordant vers la gauche le domaine où les membres de gauche ont été définis. On remplace en fait le contour $\operatorname{Re} s_{3}=\gamma$ par un contour d'intégration obtenu en joignant les points

$$
\gamma-i \infty, \gamma-i T, \gamma^{\prime}-i T, \gamma^{\prime}+i T, \gamma+i T, \gamma+i \infty
$$

où $T$ (qui dépendra de $s_{2}$ lorsque nous considèrerons $J$ ) est grand par rapport à $\operatorname{Im} m(s)$ et $\operatorname{Im} m\left(s_{2}\right)$. (La condition sur $T$ est nécessaire pour s'assurer que le contour croise bien les pôles précisés à chaque étape.) Nous aurons besoin d'être en mesure de montrer que la contribution des intégrales prises sur les deux premiers segments et les deux derniers segments sont $o(1)$; et le moyen le plus simple de le faire est d'utiliser un argument impliquant le lemme 1. Nous faisons ensuite tendre $T$ vers $+\infty$. Les manipulations analogues pour les intégrales $K_{2}$ et $K_{3}$ introduites dans la suite sont semblables quoique plus simples.

Pour que $J\left(c_{2}, c_{3}\right)$ soit bien défini, les conditions $\operatorname{Re}\left(2 s_{i}+s_{j}\right)>0$ de l'énoncé du corollaire du lemme 4 doivent être satisfaites, l'intégrande doit être fini en tout point du fermé sur lequel on intègre et la double intégrale doit être absolument convergente. Supposons pour simplifier que $c_{2}$ et $c_{3}$ sont positifs, la première condition est alors équivalente à

$$
\operatorname{Re} s>\max \left(c_{2}+\frac{1}{2} c_{3}, \frac{1}{2} c_{2}+c_{3}\right) \text {. }
$$

TOME $135-2007-\mathrm{N}^{\mathrm{O}} 1$ 
La seconde condition est

$$
n_{1} \operatorname{Re} s+\left(n_{2}-n_{1}\right) c_{2}+\left(n_{3}-n_{1}\right) c_{3} \begin{cases}\neq 1 & \text { si } b\left(n_{1}, n_{2}, n_{3}\right)>0 \\ \geqslant 1 & \text { si } b\left(n_{1}, n_{2}, n_{3}\right)<0\end{cases}
$$

où si on suppose l'hypothèse de Riemann les « $\geqslant 1 »$ dans la seconde ligne sont remplacés par « $\neq \frac{1}{2} »$. On doit aussi imposer $\operatorname{Re} s \neq c_{2}+c_{3}$, pour que le facteur $\left(s-s_{2}-s_{3}\right)$ du dénominateur de l'intégrande ne s'annule pas. Pour la troisième condition nous appliquons le lemme 1 avec les estimations

$$
\zeta(\sigma+i t)=O\left(|t|^{\mu+\varepsilon}\right) \text { où } \mu=\left\{\begin{array}{l}
\frac{1}{2}-\frac{2}{3} \sigma \text { si } 0 \leqslant \sigma \leqslant \frac{1}{2}, \\
\frac{1}{3}-\frac{1}{3} \sigma \text { si } \frac{1}{2} \leqslant \sigma \leqslant 1,
\end{array}\right.
$$

lorsque $t \geqslant 1$. Pour ces valeurs $c_{2}, c_{3}$ nous avons besoin de nous assurer que l'exposant $\alpha$ obtenu vérifie $\alpha<1$; nous nous restreignons à un demi-plan de la forme $\operatorname{Re} s>\sigma_{0}$; en fait dans la suite tous les demi-plans sur lesquels nous travaillerons l'exposant $\alpha$ maximal sera $\leqslant \frac{5}{6}+o(1)$. Nous devrons seulement décaler les contours de sorte que dans les intégrales faisant intervenir la fonction $\Psi_{5}$ dans (2.15) celle-ci soit holomorphe. Donc les seuls pôles qui nous concerneront durant l'intégration seront ceux de $\zeta\left(3 s_{j}\right)$ et $\zeta\left(2 s_{j}+s_{k}\right)$. (Cependant ce ne sera plus le cas pour les résidus $R_{j k}$.) Tout cela implique que le demi-plan sur lequel on travaille sera divisé en bandes contigües, où, dans chacune d'elles, $J\left(c_{2}, c_{3}\right)$ est holomorphe. Des remarques semblables restent pertinentes pour les intégrales $K_{3}$ et $K_{2}$ introduites en (2.20) et (2.26).

Nous prolongeons $Z(s)$ à Re $s>\frac{3}{4}$ en plusieurs étapes. Prenons $\varepsilon_{1}$ un nombre réel positif arbitraire. Nous choisissons $c_{2}=c_{3}=\frac{1}{3}+\varepsilon_{1}$, et disons que nous supposons

$$
1+4 \varepsilon_{1}<\operatorname{Re} s<\frac{13}{12} .
$$

(Le majorant ici est fixé suffisamment proche de 1 de sorte que les résultats sur les pôles rencontrés soient valables dans tout (2.17).)

(i) L'intégrale intérieure dans $J\left(c_{2}, c_{3}\right)$ étant relative à $s_{3}$, nous décalons le contour d'intégration $s_{3}$ vers $\operatorname{Re} s_{3}=\frac{1}{6}$. En faisant cela, nous croisons des pôles simples (d'une fonction de $s_{3}$ avec $s, s_{2}$ fixés) en $s_{3}=s_{3 j}$ avec

$$
s_{31}=\frac{1}{3}, s_{32}=\frac{1}{2}\left(1-s_{2}\right), s_{33}=1-2 s_{2}, s_{34}=1-s+s_{2} .
$$

Il vient

$$
J\left(\frac{1}{3}+\varepsilon_{1}, \frac{1}{3}+\varepsilon_{1}\right)=J\left(\frac{1}{3}+\varepsilon_{1}, \frac{1}{6}\right)+\sum_{j=1}^{4} K_{3}\left(\frac{1}{3}+\varepsilon_{1} ; s_{3 j}\right)
$$


où

$$
K_{3}\left(c ; s_{3 j}\right)=\frac{1}{2 \pi i} \int_{c-i \infty}^{c+i \infty} \operatorname{Res}_{s_{3}=s_{3 j}}\left\{\frac{s f\left(s-s_{2}-s_{3}, s_{2}, s_{3}\right)}{s_{2} s_{3}\left(s-s_{2}-s_{3}\right)}\right\} \mathrm{d} s_{2} .
$$

Pour chaque $j$ la méthode pour prolonger $K_{3}\left(\frac{1}{3}+\varepsilon_{1} ; s_{3 j}\right)$ est essentiellement la même. Pour un $j$ fixé, les pôles $s_{2 k}$ de l'intégrande, vu comme une fonction de $s_{2}$, apparaissent en nombre fini, où chaque $s_{2 k}$ est une fonction affine de $s$ qui prend la valeur $\frac{1}{3}$ en $s=1$. Nous décalons d'abord le contour d'intégration, par exemple jusqu'à $\operatorname{Re} s_{2}=\frac{1}{4}$. Sous la condition (2.17) le contour croise les pôles $s_{2 k}$ pour lesquels le coefficient de $s$ apparaissant dans la définition de $s_{2 k}$ n'est pas positif. Ainsi

$$
K_{3}\left(\frac{1}{3}+\varepsilon_{1} ; s_{3 j}\right)=K_{3}\left(\frac{1}{4} ; s_{3 j}\right)+\sum_{k} R_{j k}
$$

dans (2.17), où la somme porte sur les indices $k$ satisfaisant la condition évoquée ci-dessus et où les $R_{j k}$ sont les résidus en $s_{2}=s_{2 k}$ de l'intégrande dans (2.20). Lorsque $j=2,3,4$ l'intégrande dans (2.20) contient un facteur $\zeta(2 s-1)$ que nous pouvons sortir de l'intégrale. À cette manipulation près, $K_{3}\left(\frac{1}{4} ; s_{3 j}\right)$ est holomorphe dans la bande $\frac{13}{12}>\operatorname{Re} s>\frac{11}{12}$. Si nous nous restreignons à $1-4 \varepsilon_{1}>\operatorname{Re} s>\frac{11}{12}$, nous pouvons décaler le contour d'intégration vers la droite jusqu'à $\operatorname{Re} s_{2}=\frac{1}{3}-\varepsilon_{1}$. Pendant cette étape, le contour d'intégration croise les $s_{2 k}$ pour lesquels le coefficient de $s$ apparaissant dans la définition de $s_{2 k}$ est positif. Ainsi dans cette bande

$$
K_{3}\left(\frac{1}{4} ; s_{3 j}\right)=K_{3}\left(\frac{1}{3}-\varepsilon_{1} ; s_{3 j}\right)-\sum_{k} R_{j k}
$$

où la somme est restreinte aux indices $k$ ci-dessus. Ici $K_{3}\left(\frac{1}{3}-\varepsilon_{1} ; s_{3 j}\right)$ est holomorphe dans la bande $1-4 \varepsilon_{1}>\operatorname{Re} s>\frac{3}{4}+\frac{3}{2} \varepsilon_{1}$. De plus, nous montrerons que les $R_{j k}$ sont tous méromorphes dans la bande $\frac{13}{12}>\operatorname{Re} s>\frac{3}{4}$; de sorte que la réunion de (2.21) et (2.22) fournira un prolongement méromorphe de $K_{3}\left(\frac{1}{3}+\varepsilon_{1} ; s_{3 j}\right)$ jusqu'à Re $s>\frac{3}{4}+\frac{3}{2} \varepsilon_{1}$.

(ii) Pourvu que $\frac{5}{12}<\operatorname{Re} s_{1}<\frac{2}{3}$, ce qui est équivalent à

$$
\frac{11}{12}+\varepsilon_{1}<\operatorname{Re} s<\frac{7}{6}+\varepsilon_{1},
$$

la double intégrale $J\left(\frac{1}{3}+\varepsilon_{1}, \frac{1}{6}\right)$ est bien définie et holomorphe. En nous restreignant à la bande

$$
\frac{11}{12}+\varepsilon_{1}<\operatorname{Re} s<1
$$

et interchangeant l'ordre de sommation de sorte que l'intégrale intérieure porte sur $s_{2}$, nous décalons le contour d'intégration de $s_{2}$ jusqu'à $\operatorname{Re} s_{2}=\frac{1}{6}$. En 
faisant cela, nous croisons des pôles simples (d'une fonction de $s_{2}$ avec $s, s_{3}$ fixés) en $s_{2}=s_{21}$ et $s_{22}$ avec

$$
s_{21}=\frac{1}{3}, s_{22}=1-s+s_{3} .
$$

(C'est pour être sûr de croiser ce deuxième pôle que nous avons imposé la condition $\operatorname{Re} s<1$.) Ainsi nous obtenons

$$
J\left(\frac{1}{3}+\varepsilon_{1}, \frac{1}{6}\right)=J\left(\frac{1}{6}, \frac{1}{6}\right)+K_{2}\left(\frac{1}{6} ; s_{21}\right)+K_{2}\left(\frac{1}{6} ; s_{22}\right)
$$

avec

$$
K_{2}\left(c ; s_{2 j}\right)=\frac{1}{2 \pi i} \int_{c-i \infty}^{c+i \infty} \underset{s_{2}=s_{2 j}}{\operatorname{Res}}\left\{\frac{s f\left(s-s_{2}-s_{3}, s_{2}, s_{3}\right)}{s_{2} s_{3}\left(s-s_{2}-s_{3}\right)}\right\} \mathrm{d} s_{3} .
$$

Pourvu que $\frac{5}{12}<\operatorname{Re} s_{1}<\frac{2}{3}$, ce qui est cette fois-ci équivalent à

$$
\frac{3}{4}<\operatorname{Re} s<1
$$

la double intégrale $J\left(\frac{1}{6}, \frac{1}{6}\right)$ est encore bien définie et holomorphe. Utilisant (2.19) dans la bande (2.23), et la réunion de (2.19) et (2.25) dans la bande (2.27), nous avons donc réduit le problème du prolongement de $Z(s)$ jusqu'à $\operatorname{Re} s>\frac{3}{4}$ au problème correspondant pour $K_{3}$ et $K_{2}$ qui apparaissent respectivement en (2.20) et (2.26).

(iii) L'intégrande dans $K_{3}\left(\frac{1}{3}+\varepsilon_{1} ; s_{31}\right)=K_{3}\left(\frac{1}{3}+\varepsilon_{1} ; \frac{1}{3}\right)$ est

$$
\frac{s f_{1}\left(s-s_{2}-\frac{1}{3}, s_{2}, \frac{1}{3}\right)}{s_{2}\left(s-s_{2}-\frac{1}{3}\right)} \text { où } \quad f_{1}\left(s_{1}, s_{2}, s_{3}\right)=\frac{f\left(s_{1}, s_{2}, s_{3}\right)}{\zeta\left(3 s_{3}\right)} .
$$

Les seuls pôles qui nous concerne de l'intégrande vue comme une fonction de $s_{2}$ sont les pôles triples en $s_{23}=\frac{1}{3}$ et $s_{24}=s-\frac{2}{3}$ et les pôles simples $s_{25}=2 s-\frac{5}{3}$ et $s_{26}=\frac{4}{3}-s$. Si nous écrivons

$$
R_{1 j}=\operatorname{Res}_{s_{2}=s_{2 j}}\left\{\frac{s f_{1}\left(s-s_{2}-\frac{1}{3}, s_{2}, \frac{1}{3}\right)}{s_{2}\left(s-s_{2}-\frac{1}{3}\right)}\right\}
$$

alors $R_{1 j}$ est méromorphe dans $\operatorname{Re} s>\frac{1}{2}$ lorsque $j=3,4$ et dans $\frac{3}{2}>\operatorname{Re} s>\frac{3}{4}$ lorsque $j=5,6$.

Nous désignons par $g_{1}\left(s, s_{2}\right)$ l'expression entre accolades dans (2.28). Puisque $g_{1}$ vu comme une fonction de $s_{2}$ a un pôle triple $s_{23}=\frac{1}{3}$, le résidu vaut

$$
R_{13}=\lim _{s_{2} \rightarrow \frac{1}{3}} \frac{\partial^{2}}{\partial s_{2}^{2}}\left\{\frac{1}{2}\left(s_{2}-\frac{1}{3}\right)^{3} g_{1}\left(s, s_{2}\right)\right\} .
$$

On vérifie aisément que $R_{13}$ à un pôle d'ordre 7 en $s=1$ et aucun autre pôle dans $\operatorname{Re} s>\frac{3}{4}$. De même, nous avons

$$
R_{14}=\lim _{s_{2} \rightarrow s-\frac{2}{3}} \frac{\partial^{2}}{\partial s_{2}^{2}}\left\{\frac{1}{2}\left(s_{2}-s+\frac{2}{3}\right)^{3} g_{1}\left(s, s_{2}\right)\right\} .
$$


La relation $g_{1}\left(s, s_{2}\right)=g_{1}\left(s, s-s_{2}-\frac{1}{3}\right)$ fournit alors la relation

$$
R_{14}=-R_{13} \text {. }
$$

Regardons $R_{15}$ et $R_{16}$. Nous avons

$$
\begin{aligned}
R_{15} & =\lim _{s_{2} \rightarrow 2 s-\frac{5}{3}}\left\{\left(s_{2}-2 s+\frac{5}{3}\right) g_{1}\left(s, s_{2}\right)\right\} \\
& =-\frac{s f\left(s_{1}, s_{2}, s_{3}\right) / s_{1} s_{2} s_{3}}{3 \zeta\left(3 s_{3}\right) \zeta\left(2 s_{1}+s_{2}\right)} \quad \text { évalué en }\left(\frac{4}{3}-s, 2 s-\frac{5}{3}, \frac{1}{3}\right), \\
R_{16} & =\lim _{s_{2} \rightarrow \frac{4}{3}-s}\left\{\left(s_{2}+s-\frac{4}{3}\right) g_{1}\left(s, s_{2}\right)\right\} \\
& =\frac{s f\left(s_{1}, s_{2}, s_{3}\right) / s_{1} s_{2} s_{3}}{3 \zeta\left(3 s_{3}\right) \zeta\left(s_{1}+2 s_{2}\right)} \quad \text { évalué en }\left(2 s-\frac{5}{3}, \frac{4}{3}-s, \frac{1}{3}\right) .
\end{aligned}
$$

Puisque $f$ est symétrique en ses deux premiers arguments, on a $R_{15}=-R_{16}$. D'après le lemme 4 , la fonction $\Psi_{N}\left(\frac{4}{3}-s, 2 s-\frac{5}{3}, \frac{1}{3}\right)$ est holomorphe dans

$$
\left\{s \in \mathbb{C}: \frac{3}{4}+\frac{3}{4(N+1)}<\operatorname{Re} s<\frac{3}{2}-\frac{3}{2(N+1)}\right\}
$$

pourvu que $N>2$. Cela fournit un prolongement méromorphe de $R_{15}$ dans $\operatorname{Re} s>\frac{3}{4}$.

Utilisant le procédé décrit à la fin du paragraphe (i), nous avons

$$
\begin{aligned}
K_{3}\left(\frac{1}{3}+\varepsilon_{1} ; s_{31}\right) & =K_{3}\left(\frac{1}{4} ; s_{31}\right)+R_{13}+R_{16} \\
& =K_{3}\left(\frac{1}{4} ; s_{31}\right)+R_{13}-R_{15} \quad \text { dans } \frac{13}{12}>\operatorname{Re} s>1+4 \varepsilon_{1}, \\
K_{3}\left(\frac{1}{4} ; s_{31}\right) & =K_{3}\left(\frac{1}{3}-\varepsilon_{1} ; s_{31}\right)-R_{14}-R_{15} K_{3}\left(\frac{1}{3}-\varepsilon_{1} ; s_{31}\right) \\
& +R_{13}-R_{15} \quad \text { dans } 1-4 \varepsilon_{1}>\operatorname{Re} s>\frac{23}{24} .
\end{aligned}
$$

Ici $K_{3}\left(\frac{1}{4} ; s_{31}\right)$ est holomorphe dans $\frac{23}{24}<\operatorname{Re} s<\frac{13}{12}$ et $K_{3}\left(\frac{1}{3}-\varepsilon_{1} ; s_{31}\right)$ est holomorphe dans $\frac{3}{4}-\frac{1}{2} \varepsilon_{1}<\operatorname{Re} s<1-\varepsilon_{1}$.

(iv) L'intégrande dans $K_{3}\left(\frac{1}{3}+\varepsilon_{1} ; s_{32}\right)=K_{3}\left(\frac{1}{3}+\varepsilon_{1} ; \frac{1}{2}\left(1-s_{2}\right)\right)$ est

$$
\frac{s f_{2}\left(s-\frac{1}{2} s_{2}-\frac{1}{2}, s_{2}, \frac{1}{2}\left(1-s_{2}\right)\right)}{s_{2}\left(s-\frac{1}{2} s_{2}-\frac{1}{2}\right)\left(1-s_{2}\right)} \quad \text { où } \quad f_{2}\left(s_{1}, s_{2}, s_{3}\right)=\frac{f\left(s_{1}, s_{2}, s_{3}\right)}{\zeta\left(s_{2}+2 s_{3}\right)} \text {. }
$$

Nous pouvons sortir le facteur $\zeta(2 s-1)$ provenant du facteur $\zeta\left(2 s_{1}+s_{2}\right)$ de $f$ de l'intégrale. Les pôles de l'intégrande vu comme une fonction de $s_{2}$ sont un pôle triple en $s_{23}=\frac{1}{3}$ et des pôles simples en

$$
s_{25}=2 s-\frac{5}{3}, \quad s_{27}=1-\frac{2}{3} s, \quad s_{28}=\frac{2}{3} s-\frac{1}{3}, \quad s_{29}=\frac{4}{3} s-1 .
$$

Posons

$$
R_{2 j}=\operatorname{Res}_{s_{2}=s_{2 j}}\left\{\frac{s f_{2}\left(s-\frac{1}{2} s_{2}-\frac{1}{2}, s_{2}, \frac{1}{2}\left(1-s_{2}\right)\right)}{s_{2}\left(s-\frac{1}{2} s_{2}-\frac{1}{2}\right)\left(1-s_{2}\right)}\right\}
$$


et désignons par $g_{2}\left(s, s_{2}\right)$ l'expression entre accolades dans (2.33). Puisque $g_{2}$ vu comme une fonction de $s_{2}$ a un pôle triple en $s_{2}=s_{23}$, nous avons

$$
R_{23}=\lim _{s_{2} \rightarrow \frac{1}{3}} \frac{\partial^{2}}{\partial s_{2}^{2}}\left\{\frac{1}{2}\left(s_{2}-\frac{1}{3}\right)^{3} g_{2}\left(s, s_{2}\right)\right\} \text {. }
$$

Il est facile de vérifier que $R_{23}$ a un pôle d'ordre 7 en $s=1$ et pas d'autre pôle dans $\operatorname{Re} s>\frac{3}{4}$. Les autres résidus sont donnés par

$$
\begin{aligned}
& R_{25}=\lim _{s_{2} \rightarrow s_{25}}\left\{\left(s_{2}-s_{25}\right) g_{2}\left(s, s_{2}\right)\right\} \\
& =-\frac{s f\left(s_{1}, s_{2}, s_{3}\right) / s_{1} s_{2} s_{3}}{3 \zeta\left(s_{2}+2 s_{3}\right) \zeta\left(3 s_{1}\right)} \quad \quad \text { évalué en }\left(\frac{1}{3}, 2 s-\frac{5}{3}, \frac{4}{3}-s\right), \\
& R_{27}=\lim _{s_{2} \rightarrow s_{27}}\left\{\left(s_{2}-s_{27}\right) g_{2}\left(s, s_{2}\right)\right\} \\
& =\frac{s f\left(s_{1}, s_{2}, s_{3}\right) / s_{1} s_{2} s_{3}}{3 \zeta\left(s_{2}+2 s_{3}\right) \zeta\left(s_{1}+2 s_{2}\right)} \quad \quad \text { évalué en }\left(\frac{4}{3} s-1,1-\frac{2}{3} s, \frac{1}{3} s\right), \\
& R_{28}=\lim _{s_{2} \rightarrow s_{28}}\left\{\left(s_{2}-s_{28}\right) g_{2}\left(s, s_{2}\right)\right\} \\
& =-\frac{s f\left(s_{1}, s_{2}, s_{3}\right) / s_{1} s_{2} s_{3}}{3 \zeta\left(s_{2}+2 s_{3}\right) \zeta\left(s_{1}+2 s_{3}\right)} \quad \text { évalué en }\left(\frac{2}{3} s-\frac{1}{3}, \frac{2}{3} s-\frac{1}{3}, \frac{2}{3}-\frac{1}{3} s\right), \\
& R_{29}=\lim _{s_{2} \rightarrow s_{29}}\left\{\left(s_{2}-s_{29}\right) g_{2}\left(s, s_{2}\right)\right\} \\
& =-\frac{s f\left(s_{1}, s_{2}, s_{3}\right) / s_{1} s_{2} s_{3}}{3 \zeta\left(s_{2}+2 s_{3}\right) \zeta\left(2 s_{1}+s_{3}\right)} \quad \text { évalué en }\left(\frac{1}{3} s, \frac{4}{3} s-1,1-\frac{2}{3} s\right) .
\end{aligned}
$$

Interchanger $s_{1}$ et $s_{3}$ permet de montrer $R_{25}=R_{15}$. Une permutation cyclique de $s_{1}, s_{2}, s_{3}$ fournit aussi $R_{29}=-R_{27}$. La fonction $R_{2 j}$ est méromorphe dans $\operatorname{Re} s>\frac{1}{2}$ lorsque $j=3,8$, dans $2>\operatorname{Re} s>\frac{2}{3}$ lorsque $j=7,9$ et dans $\frac{3}{2}>\operatorname{Re} s>\frac{3}{4}$ lorsque $j=5$.

Étudions précisément $R_{27}$. D'après le lemme $4, \Psi_{11}\left(\frac{4}{3} s-1,1-\frac{2}{3} s, \frac{1}{3} s\right)$ est une fonction holomorphe dans $\frac{5}{4}>\operatorname{Re} s>\frac{3}{4}$. Les seuls facteurs zêta de $f$ qui peuvent fournir des pôles de $R_{27}$ dans cette bande sont ceux qui proviennent des triplets $\left(n_{1}, n_{2}, n_{3}\right)$ qui satisfont les conditions du lemme 3 et pour lesquels

$$
\operatorname{Re}\left(\left(\frac{4}{3} s-1\right) n_{1}+\left(1-\frac{2}{3} s\right) n_{2}+\frac{1}{3} s n_{3}\right) \leqslant 1
$$

est compatible avec $\frac{5}{4}>\operatorname{Re} s>\frac{3}{4}$. Si $2 n_{2}>4 n_{1}+n_{3}$, nous vérifions que cela peut seulement intervenir pour les triplets $(0,2,1)$ et $(0,3,0)$. Sinon, puisque l'inégalité (2.34) est équivalente à

$$
9\left(4 n_{1}-2 n_{2}+n_{3}\right)\left(\operatorname{Re} s-\frac{2}{3}\right) \leqslant 9-3 n_{2}-\left(2 n_{2}+2 n_{3}-n_{1}\right),
$$

de rapides calculs montrent que cela arrive seulement pour $(3,0,3),(4,1,1)$, $(4,0,2),(6,0,3)$ et les triplets pour lesquels $n_{1}+n_{2}+n_{3}=3$. 
Étudions maintenant $R_{28}$. D'après le lemme $4, \Psi_{5}\left(\frac{2}{3} s-\frac{1}{3}, \frac{2}{3} s-\frac{1}{3}, \frac{2}{3}-\frac{1}{3} s\right)$ est holomorphe dans $\operatorname{Re} s>\frac{3}{4}$; donc les seuls facteurs de $f$ qui puissent fournir des pôles à $R_{28}$ dans cette bande sont ceux qui proviennent des triplets $\left(n_{1}, n_{2}, n_{3}\right)$ vérifiant $n_{1}+n_{2}+n_{3}=3$, et ceux-ci contribuent seulement au pôle en $s=1$ d'ordre 7 .

Utilisant le procédé décrit à la fin du paragraphe (i), nous avons

$$
K_{3}\left(\frac{1}{3}+\varepsilon_{1} ; s_{32}\right)=K_{3}\left(\frac{1}{4} ; s_{32}\right)+R_{23}+R_{27}
$$

dans $\frac{13}{12}>\operatorname{Re} s>1+4 \varepsilon_{1}$, et

$$
\begin{aligned}
K_{3}\left(\frac{1}{4} ; s_{32}\right) & =K_{3}\left(\frac{1}{3}-\varepsilon_{1} ; s_{32}\right)-R_{25}-R_{28}-R_{29} \\
& =K_{3}\left(\frac{1}{3}-\varepsilon_{1} ; s_{32}\right)-R_{15}-R_{28}+R_{27}
\end{aligned}
$$

dans $1-4 \varepsilon_{1}>\operatorname{Re} s>\frac{15}{16}$. Ici $K_{3}\left(\frac{1}{4} ; s_{32}\right)$ où l'on a enlevé le facteur $\zeta(2 s-1)$ est holomorphe dans $\frac{9}{8}>\operatorname{Re} s>\frac{15}{16}$ et $K_{3}\left(\frac{1}{3}-\varepsilon_{1} ; s_{32}\right)$ avec le même facteur enlevé est holomorphe dans $1-4 \varepsilon_{1}>\operatorname{Re} s>\frac{3}{4}-\frac{3}{4} \varepsilon_{1}$.

(v) L'intégrande dans $K_{3}\left(\frac{1}{3}+\varepsilon_{1} ; s_{33}\right)=K_{3}\left(\frac{1}{3}+\varepsilon_{1} ; 1-2 s_{2}\right)$ est

$$
\frac{s f_{3}\left(s+s_{2}-1, s_{2}, 1-2 s_{2}\right)}{s_{2}\left(s+s_{2}-1\right)\left(1-2 s_{2}\right)} \quad \text { où } \quad f_{3}\left(s_{1}, s_{2}, s_{3}\right)=\frac{f\left(s_{1}, s_{2}, s_{3}\right)}{\zeta\left(2 s_{2}+s_{3}\right)} \text {. }
$$

L'intégrande contient lui aussi un facteur $\zeta(2 s-1)$, provenant cette fois-ci du facteur $\zeta\left(2 s_{1}+s_{3}\right)$ dans $f$, qui peut être sorti de l'intégrale. Les pôles de l'intégrande sont un pôle triple en $s_{23}=\frac{1}{3}$ et les pôles simples

$$
s_{26}=\frac{4}{3}-s, \quad s_{27}=1-\frac{2}{3} s, \quad s_{28}=\frac{2}{3}-\frac{1}{3} s, \quad s_{210}=\frac{1}{3} s .
$$

Notons

$$
R_{3 j}=\operatorname{Res}_{s_{2}=s_{2 j}}\left\{\frac{s f_{3}\left(s+s_{2}-1, s_{2}, 1-2 s_{2}\right)}{s_{2}\left(s+s_{2}-1\right)\left(1-2 s_{2}\right)}\right\}
$$

et $g_{3}\left(s, s_{2}\right)$ l'expression entre accolades dans (2.38). Afin de calculer les $R_{3 j}$ nous utiliserons à plusieurs reprises la relation

$$
g_{3}\left(s, s_{2}\right)=2 g_{2}\left(s, 1-2 s_{2}\right)
$$

valable pour des raisons de symétries. Il vient

$$
R_{33}=\lim _{s_{2} \rightarrow \frac{1}{3}} \frac{\partial^{2}}{\partial s_{2}^{2}}\left\{\frac{1}{2}\left(s_{2}-\frac{1}{3}\right)^{3} g_{3}\left(s, s_{2}\right)\right\}=-R_{23} .
$$

TOME $135-2007-\mathrm{N}^{\mathrm{O}} 1$ 
Il reste à déterminer la contribution des pôles simples. La relation (2.39) fournit

$$
\begin{aligned}
R_{36} & =\lim _{s_{2} \rightarrow \frac{4}{3}-s}\left\{\left(s_{2}+s-\frac{4}{3}\right) g_{3}\left(s, s_{2}\right)\right\}=-R_{25}=-R_{15}, \\
R_{37} & =\lim _{s_{2} \rightarrow 1-\frac{2}{3} s}\left\{\left(s_{2}-1+\frac{2}{3} s\right) g_{3}\left(s, s_{2}\right)\right\}=-R_{29}=R_{27}, \\
R_{38} & =\lim _{s_{2} \rightarrow \frac{2}{3}-\frac{1}{3} s}\left\{\left(s_{2}+\frac{1}{3} s-\frac{2}{3}\right) g_{3}\left(s, s_{2}\right)\right\}=-R_{28}, \\
R_{310} & =\lim _{s_{2} \rightarrow \frac{1}{3} s}\left\{\left(s_{2}-\frac{1}{3} s\right) g_{3}\left(s, s_{2}\right)\right\}=-R_{27} .
\end{aligned}
$$

La fonction $R_{3 j}$ est méromorphe dans $\operatorname{Re} s>\frac{1}{2}$ lorsque $j=3,8$, dans $2>$ Re $s>\frac{2}{3}$ lorsque $j=7,10$ et dans $\frac{3}{2}>\operatorname{Re} s>\frac{3}{4}$ lorsque $j=6$. Utilisant le procédé décrit à la fin du paragraphe (i), nous avons

$$
\begin{aligned}
K_{3}\left(\frac{1}{3}+\varepsilon_{1} ; s_{33}\right) & =K_{3}\left(\frac{1}{4} ; s_{33}\right)+R_{33}+R_{36}+R_{37}+R_{38} \\
& =K_{3}\left(\frac{1}{4} ; s_{33}\right)-R_{23}-R_{15}+R_{27}-R_{28}
\end{aligned}
$$

dans $\frac{13}{12}>\operatorname{Re} s>1+4 \varepsilon_{1}$, et

$$
K_{3}\left(\frac{1}{4} ; s_{33}\right)=K_{3}\left(\frac{1}{3}-\varepsilon_{1} ; s_{33}\right)-R_{310}=K_{3}\left(\frac{1}{3}-\varepsilon_{1} ; s_{33}\right)+R_{27}
$$

dans $1-4 \varepsilon_{1}>\operatorname{Re} s>\frac{7}{8}$. Ici $K_{3}\left(\frac{1}{4} ; s_{33}\right)$ avec le facteur $\zeta(2 s-1)$ enlevé est holomorphe dans $\frac{9}{8}>\operatorname{Re} s>\frac{7}{8}$ et $K_{3}\left(\frac{1}{3}-\varepsilon_{1} ; s_{33}\right)$ avec le même facteur enlevé est holomorphe dans $1-\varepsilon_{1}>\operatorname{Re} s>\frac{3}{4}+\frac{3}{2} \varepsilon_{1}$. Enfin, $K_{3}\left(\frac{1}{3}+\varepsilon_{1} ; s_{33}\right)$ avec le facteur $\zeta(2 s-1)$ enlevé est holomorphe dans $1-\varepsilon_{1}>\operatorname{Re} s>\frac{3}{4}-\frac{3}{2} \varepsilon_{1}$. En décalant $s_{2}$ vers la droite, on a

$$
K_{3}\left(\frac{1}{3}-\varepsilon_{1} ; s_{33}\right)=K_{3}\left(\frac{1}{3}+\varepsilon_{1} ; s_{33}\right)-R_{33}=K_{3}\left(\frac{1}{3}+\varepsilon_{1} ; s_{33}\right)+R_{23}
$$

dans le domaine $1-3 \varepsilon_{1}>\operatorname{Re} s>\frac{3}{4}+\frac{3}{2} \varepsilon_{1}$. Ainsi $K_{3}\left(\frac{1}{3}-\varepsilon_{1} ; s_{33}\right)$ admet un prolongement méromorphe dans $1-3 \varepsilon_{1}>\operatorname{Re} s>\frac{3}{4}-\frac{3}{2} \varepsilon_{1}$.

(vi) L'intégrande dans $K_{3}\left(\frac{1}{3}+\varepsilon_{1} ; s_{34}\right)=K_{3}\left(\frac{1}{3}+\varepsilon_{1} ; 1-s+s_{2}\right)$ est

$$
\frac{s f_{4}\left(2 s-2 s_{2}-1, s_{2}, 1-s+s_{2}\right)}{s_{2}\left(2 s-2 s_{2}-1\right)\left(1-s+s_{2}\right)} \text { où } f_{4}\left(s_{1}, s_{2}, s_{3}\right)=\frac{f\left(s_{1}, s_{2}, s_{3}\right)}{\zeta\left(s_{1}+2 s_{3}\right)} \text {. }
$$

L'intégrande contient encore un facteur $\zeta(2 s-1)$ provenant du facteur $\zeta\left(s_{1}+2 s_{2}\right)$ dans $f$, qui peut être sorti de l'intégrale. Les pôles de l'intégrande sont un pôle triple en $s_{24}=s-\frac{2}{3}$ et des pôles simples en

$$
s_{23}=\frac{1}{3}, \quad s_{28}=\frac{2}{3} s-\frac{1}{3}, \quad s_{29}=\frac{4}{3} s-1, \quad s_{210}=\frac{1}{3} s .
$$

Soit

$$
R_{4 j}=\operatorname{Res}_{s_{2}=s_{2 j}}\left\{\frac{s f_{4}\left(2 s-2 s_{2}-1, s_{2}, 1-s+s_{2}\right)}{s_{2}\left(2 s-2 s_{2}-1\right)\left(1-s+s_{2}\right)}\right\} .
$$


Nous désignons par $g_{4}\left(s, s_{2}\right)$ l'expression entre accolades dans (2.44). Nous utiliserons à plusieurs reprises la relation

$$
g_{4}\left(s, s_{2}\right)=2 g_{2}\left(s, 2 s-2 s_{2}-1\right)
$$

issue des relations de symétries liant les valeurs de $f$. Nous avons ainsi

$$
R_{44}=\lim _{s_{2} \rightarrow s-\frac{2}{3}} \frac{\partial^{2}}{\partial s_{2}^{2}}\left\{\frac{1}{2}\left(s_{2}-s+\frac{2}{3}\right)^{3} g_{4}\left(s, s_{2}\right)\right\}=-R_{23} \text {. }
$$

Il reste à considérer les pôles simples. La relation (2.45) fournit

$$
\begin{aligned}
R_{43} & =\lim _{s_{2} \rightarrow \frac{1}{3}}\left\{\left(s_{2}-\frac{1}{3}\right) g_{4}\left(s, s_{2}\right)\right\}=-R_{25}=-R_{15}, \\
R_{48} & =\lim _{s_{2} \rightarrow \frac{2}{3} s-\frac{1}{3}}\left\{\left(s_{2}-\frac{2}{3} s+\frac{1}{3}\right) g_{4}\left(s, s_{2}\right)\right\}=-R_{28}, \\
R_{49} & =\lim _{s_{2} \rightarrow \frac{4}{3} s-1}\left\{\left(s_{2}-\frac{4}{3} s+1\right) g_{4}\left(s, s_{2}\right)\right\}=-R_{27}, \\
R_{410} & =\lim _{s_{2} \rightarrow \frac{1}{3} s}\left\{\left(s_{2}-\frac{1}{3} s\right) g_{4}\left(s, s_{2}\right)\right\}=-R_{29}=R_{27} .
\end{aligned}
$$

Alors $R_{4 j}$ est méromorphe dans $\operatorname{Re} s>\frac{1}{2}$ lorsque $j=4,8$, dans $2>\operatorname{Re} s>\frac{2}{3}$ lorsque $j=9,10$ et dans $\frac{3}{2}>\operatorname{Re} s>\frac{3}{4}$ lorsque $j=3$. Supposant $\operatorname{Re} s>1$, nous décalons le contour d'intégration vers la droite de $\frac{1}{3}+\varepsilon_{1}$ à $\frac{1}{3}+\varepsilon_{1}+\operatorname{Re} s-1$. En faisant cela sous la condition $\operatorname{Re} s>1+3 \varepsilon_{1}$, le contour croise $s_{24}, s_{28}, s_{210}$ définis en (2.32) et avant. Un changement de variables fournit alors

$$
\begin{aligned}
K_{3}\left(\frac{1}{3}+\varepsilon_{1} ; s_{34}\right) & =K_{3}\left(\frac{1}{3}+\varepsilon_{1}+\operatorname{Re} s-1 ; s_{34}\right)-R_{44}-R_{48}-R_{410} \\
& =K_{3}\left(\frac{1}{3}+\varepsilon_{1} ; s_{33}\right)+R_{23}+R_{28}-R_{27} .
\end{aligned}
$$

Le paragraphe (v) permet de conclure.

(vii) Pour des raisons de symétries, nous avons $K_{2}\left(\frac{1}{6} ; s_{21}\right)=K_{3}\left(\frac{1}{6} ; s_{31}\right)$. Donc $K_{2}\left(\frac{1}{6} ; s_{21}\right)$ est holomorphe dans $\frac{11}{12}<\operatorname{Re} s<\frac{13}{12}$. Nous décalons le contour vers la droite jusqu'à $\frac{1}{4}$ lorsque $\operatorname{Re} s \in\left(\frac{11}{12}, 1-4 \varepsilon_{1}\right)$. Nous ne croisons aucun pôle; nous pouvons donc nous reporter au paragraphe (iii) puisque

$$
K_{2}\left(\frac{1}{6} ; s_{21}\right)=K_{3}\left(\frac{1}{4} ; s_{31}\right) \text {. }
$$

(viii) Pour des raisons de symétries, $K_{2}\left(\frac{1}{6} ; s_{22}\right)=K_{3}\left(\frac{1}{6} ; s_{34}\right)$. Utilisant l'étude du paragraphe (vi) nous pouvons décaler le contour d'intégration de $\frac{1}{6}$ à $\frac{1}{4}+\operatorname{Re} s-1$ pourvu que Re $s \in\left(\frac{15}{16}, \frac{17}{16}\right)$. Il vient dans ce domaine

$$
K_{2}\left(\frac{1}{6} ; s_{22}\right)=K_{3}\left(\frac{1}{4}+\operatorname{Re} s-1 ; s_{34}\right)=K_{3}\left(\frac{1}{4} ; s_{33}\right)
$$

où $\operatorname{Re} s \in\left(\frac{15}{16}, \frac{17}{16}\right)$. L'intégrale $K_{3}\left(\frac{1}{4}, s_{33}\right)$ avec le facteur $\zeta(2 s-1)$ enlevé est holomorphe dans $\frac{7}{8}<\operatorname{Re} s<\frac{9}{8}$. Nous pouvons donc conclure grâce au paragraphe (v). 
En reportant dans (2.19) les relations (2.25), (2.30), (2.31), (2.35), (2.36), (2.40), (2.41), (2.46), (2.47), (2.48), nous obtenons la relation

$$
\begin{aligned}
\frac{1}{4} Z(s)=3 & R_{13}-6 R_{15}+6 R_{27}-2 R_{28}+J\left(\frac{1}{6}, \frac{1}{6}\right) \\
& +2 K_{3}\left(\frac{1}{3}-\varepsilon_{1} ; s_{31}\right)+K_{3}\left(\frac{1}{3}-\varepsilon_{1} ; s_{32}\right)+3 K_{3}\left(\frac{1}{3}-\varepsilon_{1} ; s_{33}\right)
\end{aligned}
$$

Nous obtenons ainsi un prolongement méromorphe de $Z(s)$ dans la bande $\frac{3}{4}+\frac{3}{2} \varepsilon_{1}<\operatorname{Re} s<1-4 \varepsilon_{1}$.

\section{Frontière naturelle du domaine de méromorphie}

Notons

$$
\mathcal{D}(\sigma)=\{s \in \mathbb{C}: \operatorname{Re} s>\sigma\} .
$$

La formule (2.49) ne suffit pas pour établir que la droite $\operatorname{Re} s=\frac{3}{4}$ est la frontière naturelle du domaine de méromorphie de $Z(s)$. Pour montrer cela, nous commençons par déterminer quels sont les termes qui sont prolongeables à gauche de $\operatorname{Re} s=\frac{3}{4}$.

D'après le corollaire du lemme 2.3, la fonction $R_{27}$ admet un prolongement méromorphe dans le domaine $\left\{s \in \mathbb{C}: \frac{2}{3}<\operatorname{Re} s<2\right\}$. De même, les fonctions $R_{13}, R_{23}$ et $R_{28}$ admettent un prolongement méromorphe dans $\mathcal{D}\left(\frac{1}{2}\right)$.

Les fonctions $K_{3}\left(\frac{1}{3}-\varepsilon_{1} ; s_{31}\right)$ et $K_{3}\left(\frac{1}{3}-\varepsilon_{1} ; s_{33}\right)\left(\right.$ resp. $\left.K_{3}\left(\frac{1}{3}-\varepsilon_{1} ; s_{32}\right) / \zeta(2 s-1)\right)$ admettent un prolongement dans le demi-plan $\mathcal{D}\left(\frac{3}{4}-\frac{3}{4} \varepsilon_{1}\right)\left(\operatorname{resp} . \mathcal{D}\left(\frac{3}{4}-\frac{1}{2} \varepsilon_{1}\right)\right)$.

Considérons maintenant $J\left(\frac{1}{6}, \frac{1}{6}\right)$ en se plaçant dans un domaine inclus dans

$$
\frac{3}{4}<\operatorname{Re} s<\frac{11}{12}+\frac{1}{2} \varepsilon_{1} .
$$

Nous décalons la droite d'intégration de la variable $s_{2}$ vers la droite jusqu'à $\frac{1}{3}-\varepsilon_{1}$. Les pôles croisés sont

$$
s_{211}=s-\frac{1}{2}-\frac{1}{2} s_{3}, \quad s_{212}=2 s-1-2 s_{3} .
$$

Puisque $K_{2}\left(\frac{1}{6} ; s_{211}\right)=K_{3}\left(\frac{1}{6} ; s_{32}\right)$ et $K_{2}\left(\frac{1}{6} ; s_{212}\right)=K_{3}\left(\frac{1}{6} ; s_{34}\right)$, nous obtenons

$$
\begin{aligned}
J\left(\frac{1}{6}, \frac{1}{6}\right) & =J\left(\frac{1}{3}-\varepsilon_{1}, \frac{1}{6}\right)-K_{2}\left(\frac{1}{6} ; s_{211}\right)-K_{2}\left(\frac{1}{6} ; s_{212}\right) \\
& =J\left(\frac{1}{3}-\varepsilon_{1}, \frac{1}{6}\right)-K_{3}\left(\frac{1}{6} ; s_{32}\right)-K_{3}\left(\frac{1}{6} ; s_{34}\right) .
\end{aligned}
$$

De plus, la fonction $J\left(\frac{1}{3}-\varepsilon_{1}, \frac{1}{6}\right)$ est holomorphe dans $\frac{2}{3}-\varepsilon_{1}<s<\frac{5}{6}-\frac{1}{2} \varepsilon_{1}$. On décale dans l'intégrale $K_{3}\left(\frac{1}{6} ; s_{32}\right)$ l'abscisse $\frac{1}{6}$ jusqu'à $\frac{1}{3}-\varepsilon_{1}$. Lorsque $s$ satisfait (3.1), le seul pôle rencontré est $s_{28}$. Il vient

$$
K_{3}\left(\frac{1}{6} ; s_{32}\right)=K_{3}\left(\frac{1}{3}-\varepsilon_{1} ; s_{32}\right)-R_{28}
$$

ce qui fournit un prolongement méromorphe de $K_{3}\left(\frac{1}{6} ; s_{32}\right)$ dans le demi-plan $\operatorname{Re} s>\frac{3}{4}-\frac{3}{4} \varepsilon_{1}$. 
Pour étudier $K_{3}\left(\frac{1}{6} ; s_{34}\right)$, nous nous restreignons à

$$
\frac{3}{4}<\operatorname{Re} s<\frac{19}{24} .
$$

En décalant $\frac{1}{6}$ vers $\frac{11}{24}+\operatorname{Re} s-1$, nous obtenons

$$
K_{3}\left(\frac{1}{6} ; s_{34}\right)=K_{3}\left(\frac{11}{24}+\operatorname{Re} s-1 ; s_{34}\right)=K_{3}\left(\frac{1}{3}+\frac{1}{8} ; s_{33}\right)
$$

qui est prolongeable méromorphiquement dans $\left\{s \in \mathbb{C}: \frac{3}{4}-\frac{3}{16}<\operatorname{Re}(s)<\frac{7}{8}\right\}$.

En rassemblant ces résultats dans (3.2), nous obtenons un prolongement de la fonction $J\left(\frac{1}{6}, \frac{1}{6}\right)$ à gauche de $\frac{3}{4}$ jusqu'à $\frac{3}{4}-\frac{1}{2} \varepsilon_{1}$.

Reporter ces résultats dans (2.49) permet de montrer que $Z(s)+24 R_{15}(s)$ admet donc un prolongement à gauche de $\frac{3}{4}$ jusqu'à $\frac{3}{4}-\frac{1}{2} \varepsilon_{1}$. Il reste à montrer que le domaine naturel de méromorphie de $R_{15}(s)$ est celui que l'on attend. Pour cela, il suffit de considérer $\Psi_{5}\left(\frac{4}{3}-s, 2 s-\frac{5}{3}, \frac{1}{3}\right)$. D'après l'écriture (2.8), le facteur eulérien définissant $\Psi_{5}\left(\frac{4}{3}-s, 2 s-\frac{5}{3}, \frac{1}{3}\right)$ s'écrit

$$
\begin{aligned}
\psi_{5}\left(p^{-1}, \frac{4}{3}-s, 2 s-\frac{5}{3}, \frac{1}{3}\right) & \\
& =\prod_{j \in J}\left(1-p^{-1-j(s-1)}\right)\left(1+\left(1-p^{-s}\right) \sum_{j \in J} p^{-1-j(s-1)}-p^{-3 s}\right),
\end{aligned}
$$

avec $J=\{-2,-1,0,2,3,4\}$.

Notre démonstration s'inspire de [10]. Nous introduisons la fraction

$W(X, Y)=1+\left(1-X^{3} Y\right)\left(X^{6} Y^{-2}+X^{5} Y^{-1}+X^{4}+X^{2} Y^{2}+X Y^{3}+Y^{4}\right)-X^{9} Y^{3}$ de sorte que

$$
\psi_{5}\left(p^{-1}, \frac{4}{3}-s, 2 s-\frac{5}{3}, \frac{1}{3}\right)=\prod_{j \in J}\left(1-p^{-1-j(s-1)}\right) W\left(p^{-1 / 4}, p^{3 / 4-s}\right) .
$$

Le lemme suivant permet de décrire avec précision certains zéros en $s$ du facteur $W\left(p^{-1 / 4}, p^{3 / 4-s}\right)$ s'approchant par la droite de la droite verticale d'abscisse $\frac{3}{4}$.

Lemme 5. - Il existe $p_{0}$ tel que pour tout $p \geqslant p_{0}$ il existe une famille de complexes $s_{k, p}$ telle que pour tout $k \in \mathbb{Z}$ on ait $W\left(p^{-1 / 4}, p^{3 / 4-s_{k, p}}\right)=0$ et $\operatorname{Re} s_{k, p}>\frac{3}{4}$ satisfaisant l'approximation

$$
s_{k, p}-\frac{3}{4}=\frac{1-i}{4 \sqrt{2} p^{1 / 4} \log p}-\frac{3 i}{16 p^{1 / 2} \log p}-\frac{i \pi(2 k+1)}{4 \log p}+O\left(\frac{1}{p^{3 / 4} \log p}\right) .
$$

Démonstration. - Une application du théorème de Rouché permet de montrer que, pour tout $U$ au voisinage de 0 , il existe un unique $V=V_{U}$ au voisinage de $\mathrm{e}^{i \pi / 4}$ satisfaisant l'équation $W(U, V)=0$. Il est facile de montrer que

$$
-V^{4}=1+\frac{i-1}{\sqrt{2}} U+\frac{i}{4} U^{2}+O\left(U^{3}\right),
$$


à partir de la formule $W(U, V)=1+V^{4}+U V^{3}+U^{2} V^{2}+O\left(U^{3}\right)$. Prenant $(U, V)=\left(p^{-1 / 4}, p^{3 / 4-s_{p}}\right)$, nous obtenons

$$
\begin{aligned}
\left(3-4 s_{p}\right) \log p & =i \pi(2 k+1)+\log \left(1+\frac{i-1}{\sqrt{2} p^{1 / 4}}+\frac{i}{4 p^{1 / 2}}\right)+O\left(\frac{1}{p^{3 / 4}}\right) \\
& =i \pi(2 k+1)+\frac{i-1}{\sqrt{2} p^{1 / 4}}+\frac{3 i}{4 p^{1 / 2}}+O\left(\frac{1}{p^{3 / 4}}\right)
\end{aligned}
$$

avec le logarithme complexe défini par sa série entière. En écrivant $\log (1+z)=$ $z-\frac{1}{2} z^{2}+O\left(z^{3}\right)$ au voisinage de 0 , nous obtenons l'approximation annoncée. Pour $p$ suffisamment grand, nous avons bien $\operatorname{Re} s_{k, p}>\frac{3}{4}$.

Nous sommes maintenant en mesure de conclure en montrant le résultat suivant.

Corollaire . - Pour tout $\tau \in \mathbb{R}$, il existe une suite $\left\{s_{n}\right\}_{n \in \mathbb{N}}$ de complexes distincts de $\left\{s \in \mathbb{C}: \operatorname{Re} s>\frac{3}{4}\right\}$ convergeant vers $\frac{3}{4}+i \tau$ satisfaisant $R_{15}\left(s_{n}\right)=0$. En particulier, la frontière naturelle du domaine naturel de méromorphie de $R_{15}$ est la droite $\operatorname{Re} s=\frac{3}{4}$.

Démonstration. - La factorisation (2.15) de $f$ permet d'écrire

$$
\Psi_{5}\left(\frac{4}{3}-s, 2 s-\frac{5}{3}, \frac{1}{3}\right)
$$

sous la forme

$$
\prod \zeta\left(n_{1} s_{1}+n_{2} s_{2}+n_{3} s_{3}\right)^{b\left(n_{1}, n_{2}, n_{3}\right)} \Psi_{N}\left(\frac{4}{3}-s, 2 s-\frac{5}{3}, \frac{1}{3}\right)
$$

où le produit est pris sur les indices $\left(n_{1}, n_{2}, n_{3}\right)$ satisfaisant $5<n_{1}+n_{2}+n_{3} \leqslant N$ et où $\left(s_{1}, s_{2}, s_{3}\right)=\left(\frac{4}{3}-s, 2 s-\frac{5}{3}, \frac{1}{3}\right)$. La fonction $\Psi_{N}\left(\frac{4}{3}-s, 2 s-\frac{5}{3}, \frac{1}{3}\right)$ est holomorphe sur

$$
\left\{s \in \mathbb{C}: \frac{3}{4}+\frac{3}{4(N+1)}<\operatorname{Re} s<\frac{3}{2}-\frac{3}{2(N+1)}\right\}
$$

et a un facteur eulérien qui s'annule en $s_{p}=s_{k, p}$ avec $k=[-2 \tau(\log p) / \pi]$. Nous choisissons deux constantes suffisamment petites $c_{1}$ et $c_{2}$ telles que $c_{1}<c_{2}$ et que pour tout $p$ satisfaisant

$$
c_{1} \frac{N^{4}}{(\log N)^{4}}<p \leqslant c_{2} \frac{N^{4}}{(\log N)^{4}}
$$

les $s_{p}$ soient dans le domaine (3.7) et que la valeur de $k$ soit la même. Les $s_{p}$ sont donc des zéros de $\Psi_{N}\left(\frac{4}{3}-s, 2 s-\frac{5}{3}, \frac{1}{3}\right)$ et, d'après (3.4), si $N$ tend vers l'infini, les $s_{p}$ correspondant tendent vers $\frac{3}{4}+i \tau$.

Il reste à montrer que pour $N$ suffisamment grand, il existe au moins un $p$ tel que $s_{p}$ ne soit pas un pôle du produit de zêtas, disons $\Theta_{N}(s)$, apparaissant dans (3.6). 
Nous commençons par extraire une sous-suite de nombres premiers $\left\{p_{i}\right\}_{i=1}^{r}$ telle que les $s_{p_{i}}$ soient tous distincts.

Montrons que pour tout $s$ le cardinal $K$ des nombres premiers $p$ tels que $s_{p}=s$ est au plus $N /(\log N)$. Soient $p, q$ des nombres premiers distincts $p>q$ tels que $s=s_{k, p}=s_{k, q}$. Le développement du lemme 5 implique

$$
\log p=\log q+O\left(q^{-3 / 4}\right)
$$

qui fournit bien

$$
K \ll N / \log N \text {. }
$$

Le nombre $r$ de nombres premiers $p$ tels que les $s_{p}$ soient des zéros distincts dans le domaine (3.8) est donc

$$
r \gg \frac{N^{4}}{(\log N)^{5} K} \gg \frac{N^{3}}{(\log N)^{4}} .
$$

Il reste à montrer que le nombre de pôles de $\Theta_{N}(s)$ ayant une partie imaginaire comprise entre $-|\tau|-1$ et $|\tau|+1$, par exemple, est plus petit. Montrons tout d'abord que nous pouvons restreindre l'étude des pôles de $\Theta_{N}(s)$ à ceux de $\widetilde{\Theta}_{N}(s)$ qui est le produit des

$$
\zeta\left(n_{1} s_{1}+n_{2} s_{2}+n_{3} s_{3}\right)^{b\left(n_{1}, n_{2}, n_{3}\right)}
$$

avec les restrictions supplémentaires suivantes sur les indices $n_{1}, n_{2}, n_{3}$

$$
\left\{\begin{array}{l}
0 \leqslant n_{1}<4, \quad n_{2}=2 n_{3}+2 n_{1}, \\
0 \leqslant n_{1}<2, \quad n_{2}=2 n_{3}+2 n_{1}-3, \quad n_{1}+n_{3}>1 .
\end{array}\right.
$$

Pour cela, nous observons que pour les autres triplets d'indices nous avons soit $b\left(n_{1}, n_{2}, n_{3}\right)=0$ soit $\operatorname{Re}\left(n_{1} s_{1}+n_{2} s_{2}+n_{3} s_{3}\right)>1$ lorsque $\operatorname{Re} s>\frac{3}{4}$. En effet, si $b\left(n_{1}, n_{2}, n_{3}\right) \neq 0$, nous avons $n_{2} \leqslant 2 n_{1}+2 n_{3}$. Posons $r\left(n_{1}, n_{2}, n_{3}\right)=\operatorname{Re}\left(n_{1} s_{1}+n_{2} s_{2}+n_{3} s_{3}\right)=n_{1}\left(\frac{4}{3}-\operatorname{Re} s\right)+n_{2}\left(2 \operatorname{Re} s-\frac{5}{3}\right)+\frac{1}{3} n_{3}$. Si $-n_{1}+2 n_{2}<0$, alors $r\left(n_{1}, n_{2}, n_{3}\right)>1$ dès que Re $s \in\left(\frac{3}{4}, 1\right)$. Les facteurs correspondant à ces triplets $\left(n_{1}, n_{2}, n_{3}\right)$ n'ont donc pas de zéro dans le domaine qui nous intéresse. Lorsque $-n_{1}+2 n_{2}>0$, on a lorsque $\operatorname{Re} s \in\left(\frac{3}{4}, 1\right)$

$$
r\left(n_{1}, n_{2}, n_{3}\right)>\frac{1}{4} n_{1}-\frac{1}{6}\left(n_{2}-2 n_{1}-2 n_{3}\right) \geqslant \frac{1}{4} n_{1} .
$$

Si $n_{1}=2 n_{2}$, on a alors de même $r\left(n_{1}, n_{2}, n_{3}\right) \geqslant \frac{1}{2} n_{1}$. Nous pouvons donc nous restreindre au cas $0 \leqslant n_{1}<4$ et

$$
2 n_{3}-6+\frac{7}{2} n_{1}<n_{2} \leqslant 2 n_{3}+2 n_{1}
$$

car sinon $r\left(n_{1}, n_{2}, n_{3}\right)>1$ dès que $\operatorname{Re} s \in\left(\frac{3}{4}, 1\right)$. Cela permet ainsi de se restreindre à (3.10) grâce à $3 \mid n_{1}+n_{2}+n_{3}$.

Le nombre de facteurs zêta de $\widetilde{\Theta}_{N}(s)$ est $=O(N)$. Pour chacun de ses facteurs, le nombre de pôles ayant une partie imaginaire comprise entre $-|\tau|-1$ 
et $|\tau|+1$ est d'après un théorème classique de densité de zéros de la fonction zêta de Riemann $=O(N(|\tau|+1) \log (N(|\tau|+2)))$.

Le nombre de pôles, que nous cherchions à majorer, est donc $=O\left(N^{2} \log N\right)$ à $\tau$ fixé et est négligeable par rapport au minorant obtenu en (3.9). Cela achève la démonstration du corollaire.

\section{Estimation asymptotique}

4.1. Majorations de $\zeta$. - Nous rappelons les majorations classiques de $\zeta$ conditionnelles à l'hypothèse de Riemann [14].

Lemme 6. - Soient $\varepsilon>0$. Sous l'hypothèse de Riemann, on a pour tout $k \in \mathbb{N}$ la majoration uniforme lorsque $\sigma \leqslant 1-\varepsilon$

$$
\left|\zeta^{(k)}(\sigma+i \tau)\right| \ll_{\varepsilon, k}(1+|\tau|)^{\max \left\{\frac{1}{2}-\sigma, 0\right\}+\varepsilon}
$$

et la majoration uniforme lorsque $\sigma \geqslant \frac{1}{2}+\varepsilon$

$$
|\zeta(\sigma+i \tau)|^{-1} \ll_{\varepsilon}(1+|\tau|)^{\varepsilon} .
$$

Nous aurons aussi besoin du lemme suivant pour déterminer les propriétés de $R_{15}$. Plutôt que d'utiliser la factorisation (2.15) de $f\left(s_{1}, s_{2}, s_{3}\right)$ en produit de $\zeta\left(n_{1} s_{1}+n_{2} s_{2}+n_{3} s_{3}\right)^{b\left(n_{1}, n_{2}, n_{3}\right)}$ pour $N$ tendant vers l'infini, nous préférons étudier directement le résidu $R_{15}$ dans la bande verticale

$$
\left\{s \in \mathbb{C}: \frac{4}{5}+\varepsilon<\operatorname{Re} s<\frac{5}{4}-\varepsilon\right\}
$$

via (2.15) pris en $N=5$ et un calcul explicite.

Lemme 7. - Soit $\varepsilon>0$. Il existe $G_{1}$ une fonction holomorphe ne s'annulant pas et bornée sur $\left\{s \in \mathbb{C}: \frac{4}{5}+\varepsilon<\operatorname{Re} s<\frac{5}{4}-\varepsilon\right\}$ telle que dans ce domaine on ait

$$
\Psi_{5}\left(\frac{4}{3}-s, 2 s-\frac{5}{3}, \frac{1}{3}\right)=\frac{\zeta(11 s-8)}{\zeta(8 s-6) \zeta(7 s-5) \zeta(6 s-4)^{2}} G_{1}(s) .
$$

Remarque. - Posant

$$
\begin{aligned}
E(s)=\frac{s}{\left(2 s-\frac{5}{3}\right)\left(\frac{4}{3}-s\right)} \zeta(2 s- & 1) \zeta(3 s-2) \zeta(4 s-3) \\
& \times \zeta(6 s-5) \zeta(2-s) \zeta(3-2 s) \zeta(4-3 s),
\end{aligned}
$$

nous avons ainsi

$$
-R_{15}=E(s) \Psi_{5}\left(\frac{4}{3}-s, 2 s-\frac{5}{3}, \frac{1}{3}\right)=\frac{E(s) \zeta(11 s-8) G_{1}(s)}{\zeta(8 s-6) \zeta(7 s-5) \zeta(6 s-4)^{2}} .
$$


Démonstration. - On observe que dans (3.3) l'on peut restreindre la somme et le produit aux entiers $j>0$. Pour étudier $\Psi_{5}\left(\frac{1}{3}-s, 2 s+\frac{1}{3}, \frac{1}{3}\right)$, nous nous plaçons dans $\left\{s \in \mathbb{C}: \frac{4}{5}+\varepsilon \leqslant \operatorname{Re} s+1 \leqslant \frac{5}{4}-\varepsilon\right\}$. Le facteur $\psi_{5}\left(p^{-1}, \frac{1}{3}-s, 2 s+\frac{1}{3}, \frac{1}{3}\right)$ associé au premier $p$ satisfait

$$
\begin{aligned}
=\left(1-p^{-1-2 s}\right) & \left(1-p^{-1-3 s}\right)\left(1-p^{-1-4 s}\right) \\
\times & \left(1+p^{-1-2 s}+p^{-1-3 s}+p^{-1-4 s}+O\left(p^{-1-\varepsilon}\right)\right) .
\end{aligned}
$$

En développant le produit, on montre successivement qu'il est égal à

$$
\begin{gathered}
\left(1-p^{-1-3 s}\right)\left(1-p^{-1-4 s}\right)\left(1+p^{-1-3 s}+p^{-1-4 s}-p^{-2-6 s}+O\left(p^{-1-\varepsilon}\right)\right) \\
=\left(1-p^{-1-4 s}\right)\left(1+p^{-1-4 s}-2 p^{-2-6 s}-p^{-2-7 s}+O\left(p^{-1-\varepsilon}\right)\right) \\
=\left(1-2 p^{-2-6 s}-p^{-2-7 s}-p^{-2-8 s}+p^{-3-11 s}+O\left(p^{-1-\varepsilon}\right)\right) .
\end{gathered}
$$

On factorise cette dernière expression maintenant petit à petit pour reconnaître les facteurs de la fonction zêta. Le facteur eulérien est donc de la forme

$$
\left(1-p^{-2-6 s}\right)^{2}\left(1-p^{-2-7 s}\right)\left(1-p^{-2-8 s}\right)\left(1-p^{-3-11 s}\right)^{-1}\left(1+O\left(p^{-1-\varepsilon}\right)\right)
$$

ce qui permet de trouver le résultat recherché.

4.2. Démonstration du théorème 2. - Nous nous plaçons sous l'hypothèse de Riemann. La formule (2.49) et les propriétés décrites des différentes fonctions intervenant dans son membre de droite permettent d'écrire

$$
Z(s)=-24 R_{15}+R(s)
$$

où $R$ est une fonction méromorphe sur $\mathcal{D}\left(\frac{4}{5}+\varepsilon\right)$ n'ayant aucun pôle en dehors de $s=1$ et satisfaisant la majoration dans $\left\{s \in \mathcal{D}\left(\frac{4}{5}+\varepsilon\right):|s-1| \geqslant \varepsilon\right\}$

$$
R(s) \ll_{\varepsilon}|s|^{\varepsilon} .
$$

En effet, tous les $R_{j k}$ apparaissant dans le membre de droite de (2.49) hormis $R_{15}$ peuvent s'écrire comme le produit d'une fonction holomorphe bornée dans le domaine considéré avec un produit de facteur zêta dont les arguments sont de partie réelle $>\frac{1}{2}$ et $\neq 1$ si $s \neq 1$. Le lemme 6 fournit alors un majoration de $R_{j k}$ de la forme (4.5). Le même type de majoration s'obtient de la même manière pour les $K_{3}\left(\frac{1}{3}-\varepsilon_{1} ; s_{3 j}\right)$ avec $j=1,2,3$ pourvu que $\varepsilon_{1}$ soit choisi suffisamment petit.

Montrons la majoration

$$
\operatorname{card}\{P: H(P)=n\} \ll n^{2 / 3+\varepsilon} .
$$

Nous paramétrons ces points $P$ comptés par les quadruplets $\left(x_{0}, x_{1}, x_{2}, x_{3}\right)$ tels que $x_{1} x_{2} x_{3}=x_{0}^{3}$. Nous pouvons supposer les coordonnées positives et $x_{3}=n$. On peut écrit $x_{1}=y_{1}^{3} n_{1} u_{1} v_{1}^{2}$ et $x_{2}=y_{2}^{3} n_{2} u_{2} v_{2}^{2}$ où $n_{1}$ et $n_{2}$ n'ont pas de facteurs cubiques et sont des diviseurs de $n^{2}$, les $u_{i}$ et $v_{i}$ sont premiers à $n$, sans facteur 
carré et sont des diviseurs de $n^{2}$. Alors, nous devons avoir $u_{1}=v_{2}, u_{2}=v_{1}$. De plus, $y_{i} \leqslant n / z_{i} u_{i} v_{i}^{2}$. Nous en déduisons la majoration

$$
\begin{aligned}
\operatorname{card}\{P: H(P)=n\} & \ll \sum_{u, v \leqslant n} \sum_{z_{i} \mid n^{2}}\left[\frac{n}{z_{1} u v^{2}}\right]^{1 / 3}\left[\frac{n}{z_{2} u^{2} v}\right]^{1 / 3} \\
& \ll n^{2 / 3} \tau\left(n^{2}\right)^{2}(\log 2 n)^{2} \ll n^{2 / 3+\varepsilon} .
\end{aligned}
$$

La seconde formule de Perron (voir par exemple le corollaire II.2.2.1 de [13]) fournit lorsque $\kappa=1+1 / \log B$ et $T \geqslant 2$

$$
N(B)=\frac{1}{2 \pi i} \int_{\kappa-i T}^{\kappa+i T} Z(s) \frac{B^{s} \mathrm{~d} s}{s}+O\left(B^{2 / 3+\varepsilon}(1+x(\log T) / T)\right) .
$$

Nous décalons le contour jusqu'à l'abscisse $\frac{4}{5}$. Une application immédiate du théorème des résidus fournit l'existence d'un polynôme $P$ tel que

$$
\frac{1}{2 \pi i} \int_{\kappa-i T}^{\kappa+i T} R(s) \frac{B^{s} \mathrm{~d} s}{s}=B P(\log B)+O\left(B^{4 / 5+\varepsilon} T^{\varepsilon}\right) .
$$

Compte tenu de la formule (4.4) issu du lemme 7, les majorations sous l'Hypothèse de Riemann du lemme 6 fournissent lorsque $\operatorname{Re} s \in\left[\frac{4}{5}, \frac{5}{6}\right]$

$$
R_{15}(s) \zeta(8 s-6) \ll(1+|\tau|)^{13(1-\sigma)-\frac{5}{2}+\varepsilon} .
$$

En décalant l'abscisse d'intégration de $\kappa$ à $\frac{13}{16}+\varepsilon$, on voit que les pôles rencontrés sont 1 et $\frac{9}{11}$. Notons que la valeur $\frac{5}{6}$ n'est pas un pôle de $R_{15}(s)$ puisque le produit $\left(2 s-\frac{5}{3}\right) \zeta(6 s-4)$ ne s'annule pas en $s=\frac{5}{6}$. Il vient

$$
\frac{1}{2 \pi i} \int_{\kappa-i T}^{\kappa+i T} R_{15}(s) \frac{B^{s} \mathrm{~d} s}{s}=B P_{1}(\log B)-\frac{1}{24} \gamma B^{9 / 11}+O\left(B^{13 / 16+\varepsilon} T^{\varepsilon}\right)
$$

ce qui fournit la première partie du théorème 2 en prenant $T=B^{A}$ et $A$ suffisamment grand. La constante $\gamma$ est donnée par la formule

$$
\gamma=24 \operatorname{Res}_{s=9 / 11}\left(-\frac{R_{15}(s)}{s}\right)=\frac{8 E(9 / 11) G_{1}(9 / 11)}{3 \zeta(6 / 11) \zeta(8 / 11) \zeta(10 / 11)^{2}} .
$$

Nous constatons tout d'abord que $G_{1}(\sigma)>0$ lorsque $\sigma \geqslant \frac{9}{11}$ puisque c'est un produit eulérien absolument convergeant dont chaque facteur relatif à un premier $p$ ne s'annule pas. En effet, d'après (4.4), cela provient de la nonannulation de $\psi_{5}\left(p^{-1}, \frac{4}{3}-\sigma, 2 \sigma-\frac{5}{3}, \frac{1}{3}\right)$ dont on trouvera une expression en (3.3). Nous avons ainsi

$$
\begin{aligned}
& \psi_{5}\left(p^{-1}, \frac{4}{3}-\sigma, 2 \sigma-\frac{5}{3}, \frac{1}{3}\right) \\
& =\left(1-p^{-\sigma}\right) \prod_{j \in J}\left(1-p^{-1-j(\sigma-1)}\right)\left(1+\sum_{j \in J} p^{-1-j(\sigma-1)}+p^{-\sigma}+p^{-2 \sigma}\right),
\end{aligned}
$$


avec $J=\{-2,-1,0,2,3,4\}$ et il est alors facile de vérifier que tous les facteurs sont strictement positifs. La constante $\gamma$ est donc non nulle. La fonction $G_{1}(\sigma)$ lorsque $\sigma \geqslant \frac{9}{11}$ est continue, ne s'annule pas et est positive pour $\sigma>1$. La valeur $G_{1}\left(\frac{9}{11}\right)$ est donc positive. En utilisant $\zeta(\sigma)<0$ lorsque $\left.\sigma \in\right]-2,1[$, on en déduit la positivité de $\gamma$.

Supposons maintenant que tous les zéros de $\zeta(s)$ sont simples. On sait que pour tout $T$ suffisamment grand il existe $R \in[T, T+1]$ tel que

$$
\zeta(\sigma \pm i R) \gg R^{-1 / \log \log \log R} \quad\left(\sigma \geqslant \frac{1}{2}\right) .
$$

Nous appliquons le théorème des résidus au contour joignant

$$
\kappa-\frac{1}{8} i R, \quad \kappa+\frac{1}{8} i R, \quad \frac{4}{5}+\varepsilon+\frac{1}{8} i R, \quad \frac{4}{5}+\varepsilon-\frac{1}{8} i R .
$$

Les majorations sur les contours horizentaux correspondant à $\operatorname{Re} s \geqslant \frac{13}{16}$ sont assurées par (4.10) et (4.7). Lorsque $\operatorname{Re} s \in\left[\frac{4}{5}+\varepsilon, \frac{13}{16}\right]$, l'équation fonctionnelle de $\zeta$ fournit

$$
\zeta(8 s-6)^{-1} \asymp(1+|\tau|)^{8(\sigma-1)+\frac{3}{2}} \zeta(7-8 s)^{-1}
$$

et par conséquent sur la partie du contour de partie réelle $\leqslant \frac{13}{16}$ nous avons la majoration

$$
R_{15}(s) \ll(1+|\tau|)^{4-5 \sigma+\varepsilon} .
$$

Avec le choix $R=\frac{1}{8} T$, nous obtenons l'estimation

$$
\begin{aligned}
\frac{1}{2 \pi i} \int_{\kappa-i T}^{\kappa+i T}-24 R_{15}(s) \frac{B^{s} \mathrm{~d} s}{s}= & 24 B P_{1}(\log B)+\gamma B^{9 / 11} \\
& +\sum_{|\tau| \leqslant R} \beta_{\tau} B^{13 / 16+i \tau / 8}+O\left(B^{4 / 5+\varepsilon} T^{\varepsilon}\right)
\end{aligned}
$$

où les $\frac{1}{2}+i \tau$ parcourent les zéros de la fonction zêta, les $\beta_{\tau}$ ne dépendent que de $\tau$ et satisfont

$$
\beta_{\tau}=-24 \operatorname{Res}_{s=\frac{13}{16}+\frac{1}{8} i \tau}\left(\frac{R_{15}(s)}{s}\right) .
$$

On peut alors faire tendre $R$ vers $+\infty$ avec la condition

$$
|\zeta(\sigma+i R)|>R^{-1 / \log \log \log R} \quad\left(\frac{1}{2} \leqslant \sigma<1\right) .
$$

En rassemblant les estimations (4.2) et (4.6), nous achevons la démonstration du théorème 2 . 


\section{BIBLIOGRAPHIE}

[1] G. Bhowmik, D. Essouabri \& B. Lichtin - «Meromorphic Continuation of Multivariable Euler Products and Applications », à paraître au Forum Math., arXiv:math.NT/0502508.

[2] R. DE LA BRETĖCHE - «Sur le nombre de points de hauteur bornée d'une certaine surface cubique singulière ", in Nombre et répartition de points de hauteur bornée (E. Peyre, éd.), Astérisque, vol. 251, Soc. Math. France, 1998, p. 51-77.

[3] R. DE la Bretèche \& T. D. Browning - «On Manin's conjecture for singular del Pezzo surfaces of degree four, I », Michigan Mathematical Journal 55 (2007), p. 51-80.

[4] _ , «On Manin's conjecture for singular del Pezzo surfaces of degree four, II », Math. Proc. Cambridge Philos. Soc. 143 (2007), p. 579-605.

[5] R. De la Bretèche, T. D. Browning \& U. Derenthal - « On Manin's conjecture for a certain singular cubic surface», Ann. Sci. École Norm. Sup., $4^{e}$ série $\mathbf{4 0}$ (2007), p. 1-50.

[6] D. Essouabri - «Prolongements analytiques d'une classe de fonction zêta des hauteurs et applications », Bull. Soc. Math. France 133 (2005), p. 297-329.

[7] E. FouvRY - «Sur la hauteur des points d'une certaine surface cubique singulière », in Nombre et répartition de points de hauteur bornée (E. Peyre, éd.), Astérisque, vol. 251, Soc. Math. France, 1998, p. 31-49.

[8] R. Heath-Brown, R. De la Bretèche \& B. Z. Moroz - «The density of Rational Points on the cubic surface $X_{0}^{3}=X_{1} X_{2} X_{3}$ », Math. Proc. Cambridge Philos. Soc. 125 (1999), p. 385-395.

[9] P. SAlberger - « Tamagawa measures on universal torsors and points of bounded height on Fano varieties », in Nombre et répartition de points de hauteur bornée (E. Peyre, éd.), Astérisque, vol. 251, Soc. Math. France, 1998, p. 91-258.

[10] M. DU SAUtoy - «Zeta Functions of Groups and Natural Boundaries », preprint, 2000.

[11] P. Sir Swinnerton-Dyer - «Counting points on cubic surfaces II, geometric methods in algebra and number theory », (F. Bogomolov \& Y. Tschinkel, éds.), Prog. Math., vol. 235, Birkhäuser, 2004.

[12] , « A canonical height on $X_{0}^{3}=X_{1} X_{2} X_{3}$ », in Diophantine geometry, CRM Series 4, Ed. Norm., Pisa, 2007, p. 309-322.

[13] G. Tenenbaum - Introduction à la théorie analytique et probabiliste des nombres, $2^{\mathrm{e}}$ éd., Cours Spécialisé, vol. 1, Soc. Math. France, 1995. 
[14] E. C. Titchmarsh - The theory of the Riemann Zeta-function, $2^{\mathrm{e}}$ éd., Oxford, 1986. 